Front. Math. China

DOI $\operatorname{xxxxxx}$

\title{
Smoothness of Local Times and Self-Intersection Local Times of Gaussian Random Fields
}

\author{
Zhenlong CHEN ${ }^{1}$, Dongsheng WU ${ }^{2}$, Yimin XIAO ${ }^{3}$ \\ 1 School of Statistics and Mathematics, Zhejiang Gongshang University, China \\ 2 Department of Mathematical Sciences, University of Alabama in Huntsville, U.S.A. \\ 3 Department of Statistics and Probability, Michigan State University, U.S.A.
}

(C) Higher Education Press and Springer-Verlag Berlin Heidelberg 2013

\begin{abstract}
This paper is concerned with the smoothness (in the sense of MeyerWatanabe) of the local times of Gaussian random fields. Sufficient and necessary conditions for the existence and smoothness of the local times, collision local times, and self-intersection local times are established for a large class of Gaussian random fields, including fractional Brownian motions, fractional Brownian sheets and solutions of stochastic heat equations driven by spacetime Gaussian noise.
\end{abstract}

Keywords Anisotropic Gaussian field, local time, collision local time, intersection local time, self-intersection local time, chaos expansion

MSC 60G15, 60H05, 60H07

\section{Introduction}

In recent years, Malliavin calculus has been shown to be very useful in stochastic analysis of Gaussian processes [cf. Nualart (2006)]. In particular, many authors have studied the chaos expansion and smoothness in the sense of Meyer-Watanabe of local times and intersection local times of Brownian motion, fractional Brownian motion and related self-similar Gaussian processes. See Nualart and Vives (1992), Imkeller and Weisz (1995, 1999), Imkeller et al. (1995), $\mathrm{Hu}$ (2001), $\mathrm{Hu}$ and Øksendal (2002), Eddahbi and Vives (2003), $\mathrm{Hu}$ and Nualart (2005), Yan et al. (2009), Jiang and Wang (2009), Yan and Shen (2010), Chen and Yan (2011), Shen and Yan (2011), Shen el al. (2012). However, there have been only a few results on smoothness of local times of Gaussian random fields due to their more complicated dependence structures. We refer to Imkeller and Weisz $(1994,1999)$ for the case of Brownian sheet and to Eddahbi, et al. $(2005,2007)$ for results on fractional Brownian sheets.

\footnotetext{
Received xxxx; accepted xxxx

Corresponding author: Yimin XIAO, E-mail: xiao@stt.msu.edu
} 
The main purpose of this paper is to study the smoothness in the sense of Meyer-Watanabe of the local times of a large class of Gaussian random fields, including fractional Brownian sheets and solutions of stochastic heat equations driven by space-time Gaussian noise. More specifically, let $X=\left\{X(t), t \in \mathbb{R}^{N}\right\}$ be a Gaussian random field with values in $\mathbb{R}^{d}$ defined on a probability space $(\Omega, \mathscr{F}, \mathbb{P})$ by

$$
X(t)=\left(X_{1}(t), \ldots, X_{d}(t)\right), \quad \forall t \in \mathbb{R}^{N} .
$$

We will call $X$ an $(N, d)$-Gaussian random field. We assume that the coordinate fields $X_{1}, \ldots, X_{d}$ are independent copies of a real-valued, centered Gaussian random field $X_{0}=\left\{X_{0}(t), t \in \mathbb{R}^{N}\right\}$ with continuous covariance function $R(s, t)=\mathbb{E}\left[X_{0}(s) X_{0}(t)\right]$.

Let $H=\left(H_{1}, \ldots, H_{N}\right) \in(0,1)^{N}$ be a fixed vector. For $a, b \in \mathbb{R}^{N}$ with $a_{j}<b_{j}(j=1, \ldots, N)$, let $I=[a, b]:=\prod_{j=1}^{N}\left[a_{j}, b_{j}\right] \subseteq \mathbb{R}^{N}$ be the compact interval (or a rectangle). For simplicity, we will take $I=[0,1]^{N}$ throughout this paper. We further assume that $X_{0}=\left\{X_{0}(t), t \in \mathbb{R}^{N}\right\}$ satisfies the following conditions:

(C1) There exists a positive and finite constant $c_{1}$ such that

$$
\mathbb{E}\left[\left(X_{0}(s)-X_{0}(t)\right)^{2}\right] \leqslant c_{1} \sum_{j=1}^{N}\left|s_{j}-t_{j}\right|^{2 H_{j}}, \quad \forall s, t \in I .
$$

(C2) There exists a constant $c_{2}>0$ such that for all $s, t \in I$,

$$
\operatorname{Var}\left(X_{0}(t) \mid X_{0}(s)\right) \geqslant c_{2} \sum_{j=1}^{N} \min \left\{\left|s_{j}-t_{j}\right|^{2 H_{j}},\left|t_{j}\right|^{2 H_{j}}\right\} .
$$

Here $\operatorname{Var}\left(X_{0}(t) \mid X_{0}(s)\right)$ denotes the conditional variance of $X_{0}(t)$ given $X_{0}(s)$.

The class of Gaussian random fields that satisfy Conditions (C1) and (C2) is large. When $N=1$, it includes fractional Brownian motion, bi-fractional Brownian motion and related Gaussian processes. For $N \geqslant 2$, this class contains fractional Brownian sheets [cf. Ayache and Xiao (2005), Wu and Xiao (2007) for verification], solutions to stochastic heat equation driven by spacetime Gaussian noises [Mueller and Tribe (2002), Wu and Xiao (2006), Dalang, et al. (2015), Tudor and Xiao (2015)] and many more [cf. Xiao (2009)].

The purpose of this paper is to study the existence and smoothness (in the sense of Meyer-Watanabe) of the local times and the self-intersection local times of Gaussian random fields that satisfy Conditions (C1), (C2) and/or (C3) below. Our main results in Sections 2 and 3 unify and extend the previous results in the references mentioned at the beginning of the Introduction. We should also mention that Hölder regularities of local times and their applications to sample path properties of Gaussian random fields have been studied by several 
authors, including Pitt (1978), Geman and Horowitz (1980), Xiao and Zhang (2002), Ayache and Xiao (2005), Ayache, et al. (2008), Wu and Xiao (2007, 2010, 2011), Xiao (2009), Biermé et al. (2009), Chen and Xiao (2012).

The rest of this paper is organized as follows. In Section 2 we provide a sufficient and necessary condition for the existence, and a sufficient condition for the smoothness (in the sense of Meyer-Watanabe) of the local time at any level $x \in \mathbb{R}^{d}$ for a large class of Gaussian random fields. We also prove that this condition for the smoothness is also necessary for the local times at $x=0$. We then apply the conditions to prove the existence and smoothness results for the collision local times and the intersection local times for two independent anisotropic Gaussian random fields.

Section 3 is concerned with self-intersection local times. We establish a sufficient and necessary condition for the existence and smoothness of selfintersection local times on two disjoint intervals. More interestingly, we also consider the analogous problems on two intersecting intervals. We will see that the results in the intersecting cases are different from and more difficult than those in the disjoint case.

Throughout this paper, we will use $c$ to denote unspecified positive finite constants which may be different in each appearance. More specific constants are numbered as $c_{1}, c_{2}, \ldots$.

\section{Existence and smoothness of the local times}

This section is concerned with the existence and smoothness of the local times of a Gaussian random field $X$ in the sense of Meyer-Watanabe. We start by recalling the definition of Chaos expansion, which is an orthogonal decomposition of $L^{2}(\Omega, \mathbb{P})$. We refer to Nualart and Vives (1992), Meyer (1993), $\mathrm{Hu}$ (2001), Nualart (2006) and the references therein for more information.

Let $\Omega$ be the space of continuous $\mathbb{R}^{d}$-valued functions $\omega$ on $I$. Then $\Omega$ is a Banach space with respect to the sup norm. Let $\mathscr{F}$ be the Borel $\sigma$-algebra on $\Omega$. Let $\mathbb{P}$ be a probability measure on $(\Omega, \mathscr{F})$, and $\mathbb{E}$ denote the expectation on this probability space. Denote by $L^{2}(\Omega, \mathbb{P})$ the space of all real (or complex) valued functional on $\Omega$ such that

$$
\mathbb{E}\left(F^{2}\right)=\int_{\Omega}|F(\omega)|^{2} \mathbb{P}(d \omega)<\infty .
$$

Let $Y=\left\{Y_{1}(t), \ldots, Y_{d}(t), t \in I\right\}$ be an $(N, d)$-Gaussian random field, where $Y_{1}, \ldots, Y_{d}$ are $d$ independent copies of some centered, real-valued Gaussian random field $Y_{0}$ on $I$. Let $p_{n}\left(y_{1}, \ldots, y_{k}\right)$ be a polynomial of degree $n$ of $k$ variables $y_{1}, \ldots, y_{k}$. Then, for any $t^{1}, \ldots, t^{k} \in I$ and $i_{1}, \ldots, i_{k} \in\{1, \ldots, d\}$, $p_{n}\left(Y_{i_{1}}\left(t^{1}\right), \ldots, Y_{i_{k}}\left(t^{k}\right)\right)$ is called a polynomial functional of $Y$. Let $\mathscr{P}_{n}$ be the completion with respect to the $L^{2}(\Omega, \mathbb{P})$ norm of the set of all polynomials of degree less than or equal to $n$. Then $\mathscr{P}_{n}$ is a subspace of $L^{2}(\Omega, \mathbb{P})$. Let $\mathscr{C}_{n}$ be 
the orthogonal complement of $\mathscr{P}_{n-1}$ in $\mathscr{P}_{n}$. Then $L^{2}(\Omega, \mathbb{P})$ is the direct sum of $\mathscr{C}_{n}$, i.e.,

$$
L^{2}(\Omega, \mathbb{P})=\bigoplus_{n=0}^{\infty} \mathscr{C}_{n}
$$

Namely, for any functional $F \in L^{2}(\Omega, \mathbb{P})$, there exists a sequence $\left\{F_{n}\right\}_{n=0}^{\infty}$ with $F_{n} \in \mathscr{C}_{n}$, such that $F=\sum_{n=0}^{\infty} F_{n}$. This decomposition is called the chaos expansion of $F$, and $F_{n}$ is called the $n$-th chaos of $F$. Clearly,

$$
F_{0}=\mathbb{E}(F), \quad \mathbb{E}\left(|F|^{2}\right)=\sum_{n=0}^{\infty} \mathbb{E}\left(\left|F_{n}\right|^{2}\right) .
$$

In Malliavin Calculus, the space of "smooth" functions in the sense of MeyerWatanabe [cf. Watanabe (1984), Nualart (2006)] is defined by

$$
D_{1}:=\left\{F \in L^{2}(\Omega, \mathbb{P}), F=\sum_{n=0}^{\infty} F_{n} \text { and } \sum_{n=0}^{\infty} n \mathbb{E}\left(\left|F_{n}\right|^{2}\right)<\infty\right\} .
$$

For $F \in L^{2}(\Omega, \mathbb{P})$ with a chaos expansion $F=\sum F_{n}$, define the operator $\Gamma_{u}$ with $u \in[0,1]$ by

$$
\Gamma_{u} F:=\sum_{n=0}^{\infty} u^{n} F_{n}
$$

and set $\Theta_{F}(u):=\Gamma_{\sqrt{u}} F$. Clearly $\Theta_{F}(1)=F$. Define $\Phi_{\Theta_{F}}(u):=\frac{d}{d u} \mathbb{E}\left(\left|\Theta_{F}(u)\right|^{2}\right)$, we then have

$$
\Phi_{\Theta_{F}}(u)=\sum_{n=1}^{\infty} n u^{n-1} \mathbb{E}\left(\left|F_{n}\right|^{2}\right) .
$$

In the following, we provide several technical lemmas which will be useful for proving the existence and smoothness of local times. Lemma 2.1 is similar to Lemma 8.6 in Biermé, Lacaux and Xiao (2009) whose proof is elementary. Lemmas 2.2 and 2.3 are from $\mathrm{Wu}$ and Xiao (2010).

Lemma 2.1 Let $\alpha$ and $\beta$ be positive constants, then for all $A \in(0,1)$

$$
\int_{0}^{1} \frac{1}{\left(A+t^{\alpha}\right)^{\beta}} d t \asymp \begin{cases}A^{-\left(\beta-\frac{1}{\alpha}\right)} & \text { if } \alpha \beta>1 \\ \log \left(1+A^{-\frac{1}{\alpha}}\right) & \text { if } \alpha \beta=1 \\ 1 & \text { if } \alpha \beta<1 .\end{cases}
$$

In the above, $f(A) \asymp g(A)$ means that the ratio $f(A) / g(A)$ is bounded from below and above by positive constants that do not depend on $A \in(0,1)$.

Lemma 2.2 Let $\alpha$ and $\beta$ be positive constants such that $\alpha \beta \geqslant 1$. 
(i) If $\alpha \beta>1$, then there exists a constant $c_{3}>0$ whose value depends on $\alpha$ and $\beta$ only such that for all $A \in(0,1), r>0, u^{*} \in \mathbb{R}$, all integers $n \geqslant 1$ and all distinct $u_{1}, \ldots, u_{n} \in O\left(u^{*}, r\right)$ we have

$$
\int_{O\left(u^{*}, r\right)} \frac{d u}{\left(A+\min _{1 \leqslant j \leqslant n}\left|u-u_{j}\right|^{\alpha}\right)^{\beta}} \leqslant c_{3} n A^{-\left(\beta-\frac{1}{\alpha}\right)} .
$$

where $O\left(u^{*}, r\right)$ denotes a ball centered at $u^{*}$ with radius $r$.

(ii) If $\alpha \beta=1$, then for any $\kappa \in(0,1)$ there exists a constant $c_{4}>0$ whose value depends on $\alpha, \beta$ and $\kappa$ only such that for all $A \in(0,1), r>0, u^{*} \in$ $\mathbb{R}$, all integers $n \geqslant 1$ and all distinct $u_{1}, \ldots, u_{n} \in O\left(u^{*}, r\right)$ we have

$$
\int_{O\left(u^{*}, r\right)} \frac{d u}{\left(A+\min _{1 \leqslant j \leqslant n}\left|u-u_{j}\right|^{\alpha}\right)^{\beta}} \leqslant c_{4} n \log \left[e+\left(\frac{r}{n} A^{-\frac{1}{\alpha}}\right)^{\kappa}\right] .
$$

Lemma 2.3 Let $0<\beta<1$ be a constant. Then there exists a positive constant $c_{5}$ such that the following statements hold.

(i) For all $r>0, u^{*} \in \mathbb{R}$, all integers $n \geqslant 1$ and all distinct $u_{1}, \ldots, u_{n} \in$ $O\left(u^{*}, r\right)$ we have

$$
\int_{O\left(u^{*}, r\right)} \frac{d u}{\min _{1 \leqslant j \leqslant n}\left|u-u_{j}\right|^{\beta}} \leqslant c_{5} n^{\beta} r^{-(\beta-1)} .
$$

(ii) For all constants $r>0$ and $M>0$, all $u^{*} \in \mathbb{R}$, integers $n \geqslant 1$ and all distinct $u_{1}, \ldots, u_{n} \in O\left(u^{*}, r\right)$ we have

$$
\int_{O\left(u^{*}, r\right)} \log \left[e+M\left(\min _{1 \leqslant j \leqslant n}\left|u-u_{j}\right|\right)^{-\beta}\right] d u \leqslant c_{5} r \log \left[e+M\left(\frac{r}{n}\right)^{-\beta}\right] .
$$

\subsection{General results}

We will apply the following proposition and the method of its proof to study the existence and smoothness of the local times of $X$.

Proposition 2.4 Let $X=\{X(t), t \in I\}$ be an $(N, d)$-Gaussian field defined by (1.1) and assume that $X_{0}$ satisfies Conditions (C1) and (C2) with index $H \in(0,1)^{N}$. Then, for any $\gamma>0, \lambda \geqslant 0$,

$$
\int_{I^{2}} \frac{\left|\left[\mathbb{E}\left(X_{0}(s) X_{0}(t)\right)\right]\right|^{\lambda}}{\left[\operatorname{det} \operatorname{Cov}\left(X_{0}(s), X_{0}(t)\right)\right]^{\frac{\gamma}{2}}} d s d t<\infty
$$

if and only if $\sum_{\ell=1}^{N} 1 / H_{\ell}>\gamma$. 
Proof First we prove the sufficiency. By (C2) we have

$$
\operatorname{Var}\left(X_{0}(s)\right) \geqslant \operatorname{Var}\left(X_{0}(s) \mid X_{0}\left(\frac{s}{2}\right)\right) \geqslant c_{2} 2^{-2} \sum_{j=1}^{N} s_{j}^{2 H_{j}}, \quad \forall s \in I .
$$

This and the fact that

$$
\operatorname{det} \operatorname{Cov}\left(X_{0}(s), X_{0}(t)\right)=\operatorname{Var}\left(X_{0}(s)\right) \operatorname{Var}\left(X_{0}(t) \mid X_{0}(s)\right)
$$

imply

$$
\operatorname{det} \operatorname{Cov}\left(X_{0}(s), X_{0}(t)\right) \geqslant c\left(\sum_{j=1}^{N} s_{j}^{2 H_{j}}\right)\left(\sum_{j=1}^{N} \min \left\{\left|s_{j}-t_{j}\right|^{2 H_{j}}, t_{j}^{2 H_{j}}\right\}\right) .
$$

On the other hand, it follows from the Cauchy-Schwarz inequality and the continuity of the covariance function $R(s, t)$ that

$$
\left|\left[\mathbb{E}\left(X_{0}(s) X_{0}(t)\right)\right]\right|^{\lambda} \leqslant c, \quad \forall s, t \in I .
$$

Hence, for proving the sufficiency, it suffices to verify that if $\sum_{j=1}^{N} \frac{1}{H_{j}}>\gamma$, then

$$
\int_{I^{2}} \frac{d s d t}{\left[\sum_{j=1}^{N} s_{j}^{2 H_{j}}\right]^{\frac{\gamma}{2}}\left[\sum_{j=1}^{N} \min \left\{\left|s_{j}-t_{j}\right|^{2 H_{j}}, t_{j}^{2 H_{j}}\right\}\right]^{\frac{\gamma}{2}}}<\infty .
$$

To estimate the integral in (2.12), we will assume that

$$
0<H_{1} \leqslant H_{2} \leqslant \cdots \leqslant H_{N}<1
$$

and integrate in the order of $d t_{1}, \ldots, d t_{N}, d s_{1}, \ldots, d s_{N}$. When $\sum_{j=1}^{N} \frac{1}{H_{j}}>\gamma$, there exists $k \in\{1,2, \ldots, N\}$ such that

$$
\sum_{j=1}^{k-1} \frac{1}{H_{j}} \leqslant \gamma<\sum_{j=1}^{k} \frac{1}{H_{j}}
$$

Note that

$$
\begin{aligned}
& \int_{I} \frac{d t_{1} \ldots d t_{N}}{\left[\sum_{j=1}^{N} \min \left\{\left|s_{j}-t_{j}\right|^{2 H_{j}}, t_{j}^{2 H_{j}}\right\}\right]^{\frac{\gamma}{2}}} \\
& \quad \leqslant \int_{I} \frac{d t_{1} \ldots d t_{N}}{\left[\sum_{j=1}^{k} \min \left\{\left|s_{j}-t_{j}\right|^{2 H_{j}}, t_{j}^{2 H_{j}}\right\}\right]^{\frac{\gamma}{2}}} .
\end{aligned}
$$

We distinguish two cases: (i) $\sum_{j=1}^{k-1} \frac{1}{H_{j}}<\gamma<\sum_{j=1}^{k} \frac{1}{H_{j}}$ and (ii) $\sum_{j=1}^{k-1} \frac{1}{H_{j}}=\gamma<$ $\sum_{j=1}^{k} \frac{1}{H_{j}}$, and show that the last integral in (2.15) is bounded by a constant that is independent of $s \in I$. 
In Case (i), if $k=1$, then $H_{1} \gamma<1$. We apply (i) of Lemma 2.3 to derive

$$
\int_{I} \frac{d t_{1} \ldots d t_{N}}{\left[\sum_{j=1}^{k} \min \left\{\left|s_{j}-t_{j}\right|^{2 H_{j}}, t_{j}^{2 H_{j}}\right\}\right]^{\frac{\gamma}{2}}} \leqslant \int_{I} \frac{d t_{1} \ldots d t_{N}}{\left[\min \left\{\left|s_{1}-t_{1}\right|^{2 H_{1}}, t_{1}^{2 H_{1}}\right\}\right]^{\frac{\gamma}{2}}} \leqslant c_{6}
$$

as desired. If $k>1$, then $H_{1} \gamma>1$. We first apply (i) of Lemma 2.2 with $\alpha=2 H_{1}, \beta=\frac{\gamma}{2}$ and $A=\sum_{j=2}^{N} \min \left\{\left|s_{j}-t_{j}\right|^{2 H_{j}}, t_{j}^{2 H_{j}}\right\}$ to deduce that

$$
\begin{aligned}
& \int_{0}^{1} \frac{d t_{1}}{\left[\min \left\{\left|s_{1}-t_{1}\right|^{2 H_{1}}, t_{1}^{2 H_{1}}\right\}+\sum_{j=2}^{k} \min \left\{\left|s_{j}-t_{j}\right|^{2 H_{j}}, t_{j}^{2 H_{j}}\right\}\right]^{\frac{\gamma}{2}}} \\
& \leqslant \frac{c_{7}}{\left[\sum_{j=2}^{k} \min \left\{\left|s_{j}-t_{j}\right|^{2 H_{j}}, t_{j}^{2 H_{j}}\right\}\right]^{\frac{1}{2}\left(\gamma-\frac{1}{H_{1}}\right)}},
\end{aligned}
$$

where $c_{7}$ is a constant which only depends on $H_{1}$ and $\gamma$. By repeatedly using Part (i) of Lemma 2.2 as in (2.16), after $k-1$ steps, we obtain that

$$
\begin{aligned}
& \int_{I} \frac{d t_{1} \ldots d t_{N}}{\left[\sum_{j=1}^{k} \min \left\{\left|s_{j}-t_{j}\right|^{2 H_{j}}, t_{j}^{2 H_{j}}\right\}\right]^{\frac{\gamma}{2}}} \\
& \quad \leqslant c \int_{0}^{1} \frac{d t_{k}}{\left[\min \left\{\left|s_{k}-t_{k}\right|^{2 H_{k}}, t_{k}^{2 H_{k}}\right\}\right]^{\frac{1}{2}\left(\gamma-\sum_{j=1}^{k-1} \frac{1}{H_{j}}\right)}} .
\end{aligned}
$$

Notice that $H_{k}\left(\gamma-\sum_{j=1}^{k-1} \frac{1}{H_{j}}\right)<1$, by applying (i) of Lemma 2.3 to the last integral in (2.17), we see from (2.15) that in Case (i)

$$
\int_{I} \frac{d t_{1} \ldots d t_{N}}{\left[\sum_{j=1}^{N} \min \left\{\left|s_{j}-t_{j}\right|^{2 H_{j}}, t_{j}^{2 H_{j}}\right\}\right]^{\frac{\gamma}{2}}} \leqslant c_{8}
$$

Now we consider Case (ii). Notice that $k>1$ in (2.15). We integrate in order of $d t_{1}, \ldots, d t_{N}$ and repeatedly apply Part (i) of Lemma 2.2 for $k-2$ steps to get

$$
\begin{aligned}
& \int_{I} \frac{d t_{1} \ldots d t_{N}}{\left[\sum_{j=1}^{k} \min \left\{\left|s_{j}-t_{j}\right|^{2 H_{j}}, t_{j}^{2 H_{j}}\right\}\right]^{\frac{\gamma}{2}}} \\
& \leqslant c \int_{0}^{1} \int_{0}^{1} \frac{d t_{k-1} d t_{k}}{\left[\sum_{j=k-1}^{k} \min \left\{\left|s_{j}-t_{j}\right|^{2 H_{j}}, t_{j}^{2 H_{j}}\right\}\right]^{\frac{1}{2}\left(\gamma-\sum_{j=1}^{k-2} \frac{1}{H_{j}}\right)}}
\end{aligned}
$$

Note that $H_{k-1}\left(\gamma-\sum_{j=1}^{k-2} \frac{1}{H_{j}}\right)=1$. By applying (ii) of Lemma 2.2 with $A=$ 
$\min \left\{\left|s_{k}-t_{k}\right|^{2 H_{k}}, t_{k}^{2 H_{k}}\right\}$ and Part (ii) of Lemma 2.3, we derive

$$
\begin{aligned}
& \int_{0}^{1} \int_{0}^{1} \frac{d t_{k-1} d t_{k}}{\left[\sum_{j=k-1}^{k} \min \left\{\left|s_{j}-t_{j}\right|^{2 H_{j}}, t_{j}^{2 H_{j}}\right\}\right]^{\frac{1}{2}\left(\gamma-\sum_{j=1}^{k-2} \frac{1}{H_{j}}\right)}} \\
& \leqslant c \int_{0}^{1} \log \left[e+\left(\frac{1}{2}\left(\min \left\{\left|s_{k}-t_{k}\right|^{2 H_{k}}, t_{k}^{2 H_{k}}\right\}\right)^{-\frac{1}{2 H_{k-1}}}\right)^{\kappa}\right] d t_{k} \\
& \leqslant c \log \left[e+2^{H_{k}-H_{k-1}}\right]
\end{aligned}
$$

where $\kappa \in(0,1)$ is a constant and we have used the fact that $H_{k} \geqslant H_{k-1}$. It follows from (2.19) and (2.20) that (2.18) also holds in Case (ii).

Hence, by (2.12) and (2.18), we have

$$
\int_{I^{2}} \frac{d s d t}{\left[\sum_{j=1}^{N} s_{j}^{2 H_{j}}\right]^{\frac{\gamma}{2}}\left[\sum_{j=1}^{N} \min \left\{\left|s_{j}-t_{j}\right|^{2 H_{j}}, t_{j}^{2 H_{j}}\right\}\right]^{\frac{\gamma}{2}}} \leqslant c \int_{I} \frac{d s}{\left[\sum_{j=1}^{N} s_{j}^{H_{j}}\right]^{\gamma}} .
$$

It is elementary to verify, by using Lemma 2.1, that the last integral is finite provided $\sum_{j=1}^{N} \frac{1}{H_{j}}>\gamma$. This proves (2.12), and thus the sufficiency.

To prove the necessity, we prove that if $\sum_{j=1}^{N} \frac{1}{H_{j}} \leqslant \gamma$ then

$$
\int_{I^{2}} \frac{\left|\left[\mathbb{E}\left(X_{0}(s) X_{0}(t)\right)\right]\right|^{\lambda}}{\left[\operatorname{det} \operatorname{Cov}\left(X_{0}(s), X_{0}(t)\right)\right]^{\frac{\gamma}{2}}} d s d t=\infty .
$$

For $\varepsilon_{0} \in\left(0, \frac{1}{2}\right)$, let $I_{\varepsilon_{0}}:=\left[\varepsilon_{0}, 1\right]^{N}$. Eq. (2.8) and the uniform continuity of $R(s, t)$ on $I_{\varepsilon_{0}}^{2}$ imply that there exists a constant $\delta_{0}>0$ such that for all $s, t \in\left[\varepsilon_{0}, \varepsilon_{0}+\delta_{0}\right]^{N}$,

$$
\mathbb{E}\left(X_{0}(s) X_{0}(t)\right) \geqslant \frac{1}{2} \mathbb{E}\left(X_{0}^{2}(t)\right) \geqslant c_{9}>0 .
$$

On the other hand, it follows from (2.9) and Condition (C1) that for all $s, t \in I$,

$$
\operatorname{det} \operatorname{Cov}\left(X_{0}(s), X_{0}(t)\right) \leqslant c \sum_{j=1}^{N}\left|s_{j}-t_{j}\right|^{2 H_{j}}
$$

By (2.22) and (2.23), we derive

$$
\int_{I^{2}} \frac{\left|\mathbb{E}\left(X_{0}(s) X_{0}(t)\right)\right|^{\lambda}}{\left[\operatorname{det} \operatorname{Cov}\left(X_{0}(s), X_{0}(t)\right)\right]^{\frac{\gamma}{2}}} d s d t \geqslant c \int_{\left[\varepsilon_{0}, \varepsilon_{0}+\delta_{0}\right]^{2 N}} \frac{d s d t}{\left[\sum_{j=1}^{N}\left|s_{j}-t_{j}\right|^{H_{j}}\right]^{\gamma}} .
$$

By using Lemma 2.1 again, it is elementary to verify that the last integral is infinite when $\sum_{j=1}^{N} \frac{1}{H_{j}} \leqslant \gamma$. This proves the necessity of the proposition.

In the following, we consider the existence of the local time of a Gaussian random field satisfying $(\mathrm{C} 1)$ and $(\mathrm{C} 2)$. Instead of using a Fourier analytic 
argument as in Xiao (2009) [see Geman and Horowitz (1980) for a systematic account], we approximate the Dirac delta function by the heat kernel

$$
p_{\varepsilon}(x)=\frac{1}{(2 \pi \varepsilon)^{d / 2}} \exp \left(-\frac{\|x\|^{2}}{2 \varepsilon}\right), \quad x \in \mathbb{R}^{d},
$$

and let

$$
\begin{aligned}
L_{\varepsilon}(x, I, X) & =\int_{I} p_{\varepsilon}(X(s)-x) d s \\
& =\frac{1}{(2 \pi)^{d}} \int_{I} \int_{\mathbb{R}^{d}} \exp \left(i\langle\xi, X(s)-x\rangle-\frac{\varepsilon\|\xi\|^{2}}{2}\right) d \xi d s .
\end{aligned}
$$

The following is a general result on existence of local times.

Lemma 2.5 Let $Y=\left\{\left(Y_{1}(t), \ldots, Y_{d}(t)\right), t \in I\right\}$ be an $(N, d)$-Gaussian random field, where $Y_{1}, \ldots, Y_{d}$ are $d$ independent copies of a centered, real-valued Gaussian random field $Y_{0}$ on $I$. Then for any $y \in \mathbb{R}^{d}$, as $\varepsilon \rightarrow 0, L_{\varepsilon}(y, I, Y)$ converges to a limit $L(y, I, Y))$ in $L^{2}(\Omega, \mathbb{P})$ if and only if

$$
\int_{I^{2}} \exp \left(-\frac{\|y\|^{2} \mathbb{E}\left[\left(Y_{0}(s)-Y_{0}(t)\right)^{2}\right]}{\operatorname{det} \operatorname{Cov}\left(Y_{0}(t), Y_{0}(s)\right)}\right) \frac{d s d t}{\left[\operatorname{det} \operatorname{Cov}\left(Y_{0}(t), Y_{0}(s)\right)\right]^{\frac{d}{2}}}<\infty .
$$

Proof Let $I_{2 d}$ be the identity matrix of order $2 d$ and let $\Gamma_{\varepsilon, d}(s, t)=\varepsilon I_{2 d}+$ $\operatorname{Cov}(Y(s), Y(t))$. For any $y \in \mathbb{R}^{d}$ and $\varepsilon>0$, Fubini's theorem and (2.25) imply

$$
\begin{aligned}
& \mathbb{E}\left(\left|L_{\varepsilon}(y, I, Y)\right|^{2}\right)=\frac{1}{(2 \pi)^{2 d}} \int_{I^{2}} d s d t \int_{\mathbb{R}^{2 d}} e^{-\frac{\varepsilon}{2}\left(\|\xi\|^{2}+\|\eta\|^{2}\right)} \\
& \times \mathbb{E} \exp (i\langle\xi, Y(s)-y\rangle-i\langle\eta, Y(t)-y\rangle) d \xi d \eta \\
& \left.=\frac{1}{(2 \pi)^{2 d}} \int_{I^{2}} d s d t \int_{\mathbb{R}^{2 d}} e^{-i\langle\xi-\eta, y\rangle}\right) \exp \left(-\frac{1}{2}(\xi, \eta) \Gamma_{\varepsilon, d}(s, t)(\xi, \eta)^{T}\right) d \xi d \eta \\
& =\frac{1}{(2 \pi)^{2 d}} \int_{I^{2}} \exp \left(-\frac{1}{2}(y, y) \Gamma_{\varepsilon, d}^{-1}(s, t)(y, y)^{T}\right) \frac{d s d t}{\sqrt{\operatorname{det} \Gamma_{\varepsilon, d}(s, t)}}
\end{aligned}
$$

Since the coordinate processes of $Y$ are independent copies of $Y_{0}$, we have

$$
\operatorname{det} \Gamma_{\varepsilon, d}(s, t)=\left[\operatorname{det} \Gamma_{\varepsilon, 1}(s, t)\right]^{d}
$$

and

$$
\left.\frac{1}{2}(y, y) \Gamma_{\varepsilon, d}^{-1}(s, t)\right)(y, y)^{T}=\frac{\|y\|^{2}\left(2 \varepsilon+\mathbb{E}\left[\left(Y_{0}(s)-Y_{0}(t)\right)^{2}\right]\right)}{\operatorname{det} \Gamma_{\varepsilon, 1}(s, t)},
$$

where $\Gamma_{\varepsilon, 1}(s, t)=\varepsilon I_{2}+\operatorname{Cov}\left(Y_{0}(s), Y_{0}(t)\right)$. It follows from (2.28), (2.29) and the dominated convergence theorem that

$$
\begin{aligned}
& \lim _{\varepsilon \rightarrow 0} \mathbb{E}\left(\left|L_{\varepsilon}(y, I, Y)\right|^{2}\right) \\
& =\frac{1}{(2 \pi)^{2 d}} \int_{I^{2}} \exp \left(-\frac{\|y\|^{2} \mathbb{E}\left[\left(Y_{0}(s)-Y_{0}(t)\right)^{2}\right]}{\operatorname{det} \Gamma_{0,1}(s, t)}\right) \frac{d s d t}{\left[\operatorname{det} \Gamma_{0,1}(s, t)\right]^{d / 2}} .
\end{aligned}
$$


Next we show that $\left\{L_{\varepsilon}(y, I, Y), \varepsilon>0\right\}$ is a Cauchy sequence in $L^{2}(\Omega, \mathbb{P})$ if and only if (2.26) holds. For all integers $m, n \geqslant 1$, we assume, without loss of generality, that $m=n+p$ for some integer $p$. Let $\Gamma_{n+p}(s, t)=(n+p)^{-1} I_{2 d}+$ $\operatorname{Cov}(Y(s), Y(t)), \Gamma_{n}(s, t)=n^{-1} I_{2 d}+\operatorname{Cov}(Y(s), Y(t))$ and

$$
\Gamma_{m+p, n}(s, t)=\left(\begin{array}{cc}
(m+p)^{-1} I_{d} & 0 \\
0 & n^{-1} I_{d}
\end{array}\right)+\operatorname{Cov}(Y(s), Y(t)) .
$$

Then, it follows from Fubini's theorem and (2.25) that

$$
\begin{gathered}
\mathbb{E}\left[\left(L_{\frac{1}{n+p}}(y, I, Y)-L_{\frac{1}{n}}(y, I, Y)\right)^{2}\right]=\frac{1}{(2 \pi)^{2 d}} \int_{I^{2}} d s d t \int_{\mathbb{R}^{2 d}} e^{-i\langle\xi-\eta, y\rangle} \\
\times\left\{\exp \left(-\frac{1}{2}(\xi, \eta) \Gamma_{n+p}(s, t)(\xi, \eta)^{T}\right)+\exp \left(-\frac{1}{2}(\xi, \eta) \Gamma_{n}(s, t)(\xi, \eta)^{T}\right)\right. \\
=\frac{1}{(2 \pi)^{2 d}} \int_{I^{2}}\left\{\frac{1}{\sqrt{\operatorname{det} \Gamma_{n+p}(s, t)}} \exp \left(-\frac{1}{2}(y, y) \Gamma_{n+p}^{-1}(s, t)(y, y)^{T}\right)\right. \\
+\frac{1}{\sqrt{\operatorname{det} \Gamma_{n}(s, t)}} \exp \left(-\frac{1}{2}(y, y) \Gamma_{n}^{-1}(s, t)(y, y)^{T}\right) \\
\left.\quad-\frac{2}{\sqrt{\operatorname{det} \Gamma_{n+p, n}(s, t)}} \exp \left(-\frac{1}{2}(y, y) \Gamma_{n+p, n}^{-1}(s, t)(y, y)^{T}\right)\right\} d s d t .
\end{gathered}
$$

Similarly to (2.30), we can verify that

$$
\lim _{n \rightarrow \infty} \mathbb{E}\left[\left(L_{\frac{1}{n+p}}(y, I, Y)-L_{\frac{1}{n}}(y, I, Y)\right)^{2}\right]=0
$$

if and only if (2.26) holds. Then $\left\{L_{\varepsilon}(y, I, Y), \varepsilon>0\right\}$ is a Cauchy sequence in $L^{2}(\Omega, \mathbb{P})$ if and only if that (2.26) holds. This finishes the proof.

Now we provided a sufficient and necessary condition for the existence of the local time of $X$, which complements Theorem 8.1 of Xiao (2009) and Theorem 3.1 of Jiang and Wang (2009).

Theorem 2.6 Let $X=\{X(t), t \in I\}$ be an $(N, d)$-Gaussian random field defined by (1.1) and assume that $X_{0}$ has mean zero, continuous covariance function and satisfies Conditions (C1) and (C2) with index $H \in(0,1)^{N}$. Then, for every $x \in \mathbb{R}^{d}, L_{\varepsilon}(x, I, X)$ converges in $L^{2}(\Omega, \mathbb{P})$ sense, to a limit $L(x, I, X)$ as $\varepsilon \rightarrow 0$ if and only if $\sum_{j=1}^{N} 1 / H_{j}>d$.

Proof By Lemma 2.5, we only need to verify that for any $x \in \mathbb{R}^{d}$,

$$
\mathscr{M}:=\int_{I^{2}} \exp \left(-\frac{\|x\|^{2} \mathbb{E}\left[\left(X_{0}(s)-X_{0}(t)\right)^{2}\right]}{\operatorname{det} \operatorname{Cov}\left(X_{0}(t), X_{0}(s)\right)}\right) \frac{d s d t}{\left[\operatorname{det} \operatorname{Cov}\left(X_{0}(t), X_{0}(s)\right)\right]^{\frac{d}{2}}}
$$


is finite if and only if $\sum_{j=1}^{N} \frac{1}{H_{j}}>d$.

The sufficiency follows immediately from Proposition 2.4. To prove the necessity, we derive from (2.8), (C1) and (C2) that, for any $\varepsilon_{0} \in(0,1)$, there exist constants $c_{11} \geqslant 1$ and $c_{12}>0$ such that $c_{11}^{-1} \leqslant \operatorname{Var}\left(X_{0}(s)\right) \leqslant c_{11}$ and

$$
\operatorname{Var}\left(X_{0}(t) \mid X_{0}(s)\right) \geqslant c_{12} \sum_{j=1}^{N}\left|s_{j}-t_{j}\right|^{2 H_{j}}
$$

for all $s, t \in\left[\varepsilon_{0}, 1\right]^{N}$. These inequalities and (2.9) imply

$$
\frac{\mathbb{E}\left[\left(X_{0}(s)-X_{0}(t)\right)^{2}\right]}{\operatorname{det} \Gamma_{0,1}(s, t)} \asymp 1
$$

for all $s, t \in\left[\varepsilon_{0}, 1\right]^{N}$. It follows from (2.31) that

$$
\mathscr{M} \geqslant c \int_{\left[\varepsilon_{0}, 1\right]^{2 N}} \frac{d s d t}{\left[\operatorname{det} \operatorname{Cov}\left(X_{0}(s), X_{0}(t)\right)\right]^{d / 2}} .
$$

From the proof of Proposition 2.4 with $\gamma=d$ and $\lambda=0$ we see that the last integral is infinite if $\sum_{j=1}^{N} \frac{1}{H_{j}} \leqslant d$. This proves the necessity and hence the theorem.

In order to study the smoothness of the local times, we will make use of the following lemmas. Lemma 2.7 is from $\mathrm{Hu}$ (2001), and Lemma 2.8 is from Chen and Yan (2011).

Lemma 2.7 Let $F \in L^{2}(\Omega, \mathbb{P})$. Then $F \in D_{1}$ if and only if $\Phi_{\Theta}(1)<\infty$.

Lemma 2.8 For any $d \in \mathbb{N}$, we have for $x \in[-1,1)$,

$$
\sum_{n=1}^{\infty} \sum_{\substack{k_{1}, \ldots, k_{d}=0 \\ k_{1}+\cdots+k_{d}=n}}^{n} \frac{2 n\left(2 k_{1}-1\right) ! ! \cdots\left(2 k_{d}-1\right) ! !}{\left(2 k_{1}\right) ! ! \cdots\left(2 k_{d}\right) ! !} x^{n} \asymp x(1-x)^{-\left(\frac{d}{2}+1\right)}
$$

Recall that the Hermite polynomial of degree $n$ is defined by

$$
H_{n}(x)=\frac{(-1)^{n}}{n !} e^{\frac{x^{2}}{2}} \frac{d^{n}}{d x^{n}}\left(e^{-\frac{x^{2}}{2}}\right), \quad n \in \mathbb{Z}_{+} .
$$

It is known that [cf. Nualart (2006)] for any centered Gaussian random vector $(\xi, \eta)$ with $\mathbb{E}\left(\xi^{2}\right)=\mathbb{E}\left(\eta^{2}\right)=1$, we have

$$
\mathbb{E}\left[H_{n}(\xi) H_{m}(\eta)\right]=\left\{\begin{array}{cc}
0, & m \neq n, \\
\frac{1}{n !}[\mathbb{E}(\xi \eta)]^{n}, & m=n
\end{array}\right.
$$

and for all $z \in \mathbb{C}$ and $x \in \mathbb{R}$,

$$
e^{z x-\frac{z^{2}}{2}}=\sum_{n=0}^{\infty} z^{n} H_{n}(x)
$$

We will make use of the following lemma. 
Lemma 2.9 Let $Y=\left\{\left(Y_{1}(t), \ldots, Y_{d}(t)\right), t \in I\right\}$ be an $(N, d)$-Gaussian random field, where $Y_{1}, \ldots, Y_{d}$ are $d$ independent copies of a centered, real-valued Gaussian random field $Y_{0}$ on $I$. Suppose that its local time $L(y, I, Y) \in L^{2}(\Omega, \mathbb{P})$. Then,

(i) $L(0, I, Y) \in D_{1}$ if and only if

$$
\int_{I^{2}} \frac{\left[\mathbb{E}\left(Y_{0}(s) Y_{0}(t)\right)\right]^{2}}{\left[\operatorname{det} \operatorname{Cov}\left(Y_{0}(t), Y_{0}(s)\right)\right]^{\frac{d}{2}+1}} d s d t<\infty .
$$

(ii) If 2.34) holds, then $L(y, I, Y) \in D_{1}$ for every $y \in \mathbb{R}^{d} \backslash\{0\}$.

Proof The proof is similar to that of Lemma 3.2 in Chen and Yan (2011), see also $\mathrm{Hu}(2001)$. Let $L_{\varepsilon}(y, I, Y)$ be as in (2.25) (by replacing $X$ by $Y$ ). Thanks to (2.25) and (2.33), we can write

$$
\begin{aligned}
L_{\varepsilon}(y, I, Y)= & \frac{1}{(2 \pi)^{d}} \int_{I} \int_{\mathbb{R}^{d}} e^{-i\langle\xi, y\rangle} \exp \left(i\langle\xi, Y(s)\rangle-\varepsilon \frac{\|\xi\|^{2}}{2}\right) d \xi d s \\
= & \frac{1}{(2 \pi)^{d}} \int_{I} \int_{\mathbb{R}^{d}} e^{-i\langle\xi, y\rangle} \exp \left(-\frac{1}{2}\left(\mathbb{E}\left(Y_{0}^{2}(s)\right)+\varepsilon\right)\|\xi\|^{2}\right) \\
& \quad \times \sum_{n=0}^{\infty} i^{n}\left(\mathbb{E}\left(Y_{0}^{2}(s)\right)\|\xi\|^{2}\right)^{n / 2} H_{n}\left(\frac{\langle\xi, Y(s)\rangle}{\sqrt{\mathbb{E}\left(Y_{0}^{2}(s)\right)\|\xi\|^{2}}}\right) d \xi d s \\
:= & \sum_{n=0}^{\infty} F_{n}^{y, \varepsilon} .
\end{aligned}
$$

Denote $\Phi_{\Theta_{y, \varepsilon}}(u)=\mathbb{E}\left(\left|\Gamma_{\sqrt{u}} L_{\varepsilon}(y, I, Y)\right|^{2}\right)$ and $\Phi_{\Theta_{y}}(u)=\mathbb{E}\left(\left|\Gamma_{\sqrt{u}} L(y, I, Y)\right|^{2}\right)$. Also, for simplicity of notation, let $a^{2}=\mathbb{E}\left(Y_{0}^{2}(s)\right)+\varepsilon$ and $b^{2}=\mathbb{E}\left(Y_{0}^{2}(t)\right)+\varepsilon$. It follows from (2.35) and (2.32) that

$$
\begin{aligned}
& \Phi_{\Theta_{y, \varepsilon}}(1)=\sum_{n=0}^{\infty} n \mathbb{E}\left(\left|F_{n}^{y, \varepsilon}\right|^{2}\right) \\
& =\sum_{n=0}^{\infty} \frac{n}{(2 \pi)^{2 d}} \mathbb{E}\left[\int_{I^{2}} \int_{\mathbb{R}^{2 d}} e^{-i\langle\xi-\eta, y\rangle}\left[\mathbb{E}\left(Y_{0}^{2}(s)\right) \mathbb{E}\left(Y_{0}^{2}(t)\right)\|\xi\|^{2}\|\eta\|^{2}\right]^{n / 2}\right. \\
& \quad \times \exp \left(-\frac{1}{2}\left[a^{2}\|\xi\|^{2}+b^{2}\|\eta\|^{2}\right]\right) \\
& \left.\quad \times H_{n}\left(\frac{\langle\xi, Y(s)\rangle}{\sqrt{\mathbb{E}\left(Y_{0}^{2}(s)\right)\|\xi\|^{2}}}\right) H_{n}\left(\frac{\langle\eta, Y(t)\rangle}{\sqrt{\mathbb{E}\left(Y_{0}^{2}(t)\right)\|\eta\|^{2}}}\right) d \xi d \eta d s d t\right] \\
& =\sum_{n=1}^{\infty} \frac{1}{(2 \pi)^{2 d}(n-1) !} \int_{I^{2}}\left[\mathbb{E}\left(Y_{0}(s) Y_{0}(t)\right]^{n} d s d t\right. \\
& \quad \times \int_{\mathbb{R}^{2 d}} e^{-i\langle\xi-\eta, y\rangle}\langle\xi, \eta\rangle^{n} \exp \left(-\frac{1}{2}\left[a^{2}\|\xi\|^{2}+b^{2} \mid \eta \|^{2}\right]\right) d \xi d \eta .
\end{aligned}
$$


If $y=0$, then the integrals in (2.36) become 0 for all odd numbers $n$. Hence

$$
\begin{aligned}
\Phi_{\Theta_{0, \varepsilon}}(1)= & \sum_{n=1}^{\infty} \frac{1}{(2 \pi)^{2 d}(2 n-1) !} \int_{I^{2}}\left[\mathbb{E}\left(Y_{0}(s) Y_{0}(t)\right)\right]^{2 n} d s d t \\
& \times \int_{\mathbb{R}^{2 d}}\langle\xi, \eta\rangle^{2 n} \exp \left(-\frac{1}{2}\left[a^{2}\|\xi\|^{2}+b^{2}\|\eta\|^{2}\right]\right) d \xi d \eta .
\end{aligned}
$$

By using the fact that for $k \in \mathbb{Z}_{+}, \gamma>0$,

$$
\int_{\mathbb{R}} v^{2 k} \exp \left(-\frac{\gamma v^{2}}{2}\right) d v=\sqrt{2 \pi}(2 k-1) ! ! \gamma^{-(k+1 / 2)}
$$

and the same argument as in Chen and Yan (2011, p. 1010), we obtain

$$
\begin{aligned}
& \frac{1}{(2 \pi)^{d}} \int_{\mathbb{R}^{2 d}} \frac{\langle\xi, \eta\rangle^{2 n}}{(2 n-1) !} \exp \left(-\frac{1}{2}\left[a^{2}\|\xi\|^{2}+b^{2}\|\eta\|^{2}\right]\right) d \xi d \eta \\
& =\sum_{\substack{k_{1}, \ldots, k_{d}=0 \\
k_{1}+\cdots+k_{d}=n}}^{n} \frac{2 n\left(2 k_{1}-1\right) ! ! \cdots\left(2 k_{d}-1\right) ! !}{\left(2 k_{1}\right) ! ! \cdots\left(2 k_{d}\right) ! !\left[\left(\mathbb{E}\left(Y_{0}^{2}(s)\right)+\varepsilon\right)\left(\mathbb{E}\left(Y_{0}^{2}(t)\right)+\varepsilon\right)\right]^{n+\frac{d}{2}}} .
\end{aligned}
$$

[This can be verified by using induction.] Combining (2.37) and (2.38), and applying Lemma 2.8 and the monotone convergence theorem, we obtain

$$
\begin{aligned}
& \sum_{n=1}^{\infty} \frac{1}{(2 n-1) !} \int_{I^{2}}\left[\mathbb{E}\left(Y_{0}(s) Y_{0}(t)\right)\right]^{2 n} d s d t \int_{\mathbb{R}^{2 d}}\langle\xi, \eta\rangle^{2 n} \\
& \quad \times \exp \left(-\frac{1}{2}\left[a^{2}\|\xi\|^{2}+b^{2}\|\eta\|^{2}\right]\right) d \xi d \eta \\
& \asymp \int_{I^{2}} \frac{\left[\mathbb{E}\left(Y_{0}(s) Y_{0}(t)\right)\right]^{2}}{\left\{a^{2} b^{2}-\left[\mathbb{E}\left(Y_{0}(s) Y_{0}(t)\right)\right]^{2}\right\}^{\frac{d}{2}+1}} d s d t \\
& \stackrel{\text { as } \varepsilon \rightarrow 0}{\longrightarrow} \int_{I^{2}} \frac{\left[\mathbb{E}\left(Y_{0}(s) Y_{0}(t)\right)\right]^{2}}{\left\{\mathbb{E}\left(Y_{0}^{2}(s)\right) \mathbb{E}\left(Y_{0}^{2}(t)\right)-\left[\mathbb{E}\left(Y_{0}(s) Y_{0}(t)\right)\right]^{2}\right\}^{\frac{d}{2}+1}} d s d t \\
& =\int_{I^{2}} \frac{\left[\mathbb{E}\left(Y_{0}(t) Y_{0}(s)\right)\right]^{2}}{\left[\operatorname{detcov}\left(Y_{0}(s), Y_{0}(t)\right)\right]^{\frac{d}{2}+1}} d s d t,
\end{aligned}
$$

which proves Part (i) of Lemma 2.9, thanks to Lemma 2.7.

Now we prove Part (ii) of the lemma. Notice that for $y \in \mathbb{R}^{d} \backslash\{0\}$, it does not seem easy to compute the integrals in the last equality of (2.36) explicitly. So we turn to the following upper bound.

$$
\begin{aligned}
\Phi_{\Theta_{y, \varepsilon}}(1) \leqslant & \sum_{n=1}^{\infty} \frac{1}{(2 \pi)^{2 d}(n-1) !} \int_{I^{2}}\left|\mathbb{E}\left(Y_{0}(s) Y_{0}(t)\right)\right|^{n} d s d t \\
& \times \int_{\mathbb{R}^{2 d}}|\langle\xi, \eta\rangle|^{n} \exp \left(-\frac{1}{2}\left[a^{2}\|\xi\|^{2}+b^{2}\|\eta\|^{2}\right]\right) d \xi d \eta .
\end{aligned}
$$


The sum over even integers in (2.40) is the same as in (2.39). So we only need to consider the terms over odd integers. For this purpose, let

$$
\begin{aligned}
J_{2 n+1}= & \frac{1}{(2 n) !} \int_{I^{2}}\left|\mathbb{E}\left(Y_{0}(s) Y_{0}(t)\right)\right|^{2 n+1} d s d t \\
& \times \int_{\mathbb{R}^{2 d}}|\langle\xi, \eta\rangle|^{2 n+1} \exp \left(-\frac{1}{2}\left[a^{2}\|\xi\|^{2}+b^{2}\|\eta\|^{2}\right]\right) d \xi d \eta .
\end{aligned}
$$

By using the Cauchy-Schwarz inequality and the elementary inequality

$$
x e^{-\frac{\beta}{2 n} x^{2}} \leqslant \sqrt{\frac{n}{e \beta}}, \quad \forall \beta>0 \text { and } x>0,
$$

we have

$$
|\langle\xi, \eta\rangle| e^{-\frac{1}{2 n}\left[a^{2}\|\xi\|^{2}+b^{2}\|\eta\|^{2}\right]} \leqslant \frac{n}{e a b} .
$$

Plugging this into (2.41) yields

$$
\begin{aligned}
J_{2 n+1} \leqslant & \frac{1}{2 e(2 n-1) !} \int_{I^{2}}\left|\mathbb{E}\left(Y_{0}(s) Y_{0}(t)\right)\right|^{2 n} d s d t \\
& \times \int_{\mathbb{R}^{2 d}}|\langle\xi, \eta\rangle|^{2 n} \exp \left(-\frac{n-1}{2 n}\left[a^{2}\|\xi\|^{2}+b^{2}\|\eta\|^{2}\right]\right) d \xi d \eta .
\end{aligned}
$$

The same argument for (2.38) gives

$$
\begin{aligned}
& \int_{\mathbb{R}^{2 d}} \frac{|\langle\xi, \eta\rangle|^{2 n}}{(2 n-1) !} \exp \left(-\frac{n-1}{2 n}\left[a^{2}\|\xi\|^{2}+b^{2}\|\eta\|^{2}\right]\right) d \xi d \eta \\
& =\frac{(2 \pi)^{d}}{\left(1-n^{-1}\right)^{n+\frac{d}{2}}} \sum_{\substack{k_{1}, \ldots, k_{d}=0 \\
k_{1}+\cdots+k_{d}=n}}^{n} \frac{2 n\left(2 k_{1}-1\right) ! ! \cdots\left(2 k_{d}-1\right) ! !}{\left(2 k_{1}\right) ! ! \cdots\left(2 k_{d}\right) ! !\left[a^{2} b^{2}\right]^{n+\frac{d}{2}}} .
\end{aligned}
$$

Combining (2.41)-(2.43) with (2.40), and using the same argument as in (2.39), we derive $\Phi_{\Theta_{y}}(1)<\infty$ under (2.34). This finishes the proof of Part (ii).

The following is the main theorem of this section.

Theorem 2.10 Let $X=\{X(t), t \in I\}$ be an $(N, d)$-Gaussian field defined by (1.1) and assume that $X_{0}$ satisfies (C1) and (C2) with index $H \in(0,1)^{N}$. Then the following statements hold:

(i) $L(0, I, X) \in D_{1}$ if and only if $\sum_{j=1}^{N} \frac{1}{H_{j}}>d+2$.

(ii) If $\sum_{j=1}^{N} \frac{1}{H_{j}}>d+2$, then $L(x, I, X) \in D_{1}$ for every $x \in \mathbb{R}^{d} \backslash\{0\}$.

Proof By Theorem 2.6 and Lemma 2.9, it is sufficient for us to verify that

$$
\int_{I^{2}} \frac{\left[\mathbb{E}\left(X_{0}(s) X_{0}(t)\right)\right]^{2}}{\left[\operatorname{det} \operatorname{Cov}\left(X_{0}(t), X_{0}(s)\right)\right]^{\frac{d}{2}+1}} d s d t<\infty
$$


if and only if $\sum_{j=1}^{N} \frac{1}{H_{j}}>d+2$. This follows from Proposition 2.4 with $\gamma=d+2$ and $\lambda=2$ immediately.

Remark 2.11 As we mentioned in Introduction, the class of Gaussian random fields that satisfy (C1) and (C2) is large, including fractional Brownian sheets and the solutions to stochastic heat equation driven by various space-time Gaussian noises. In particular, Theorem 2.10 recovers Theorem 11 in Eddahbi, et al. (2005) and Theorem 2.1 with $\alpha=1$ in Eddahbi and Vives (2003).

In the following we apply Theorems 2.6 and 2.10 to study the collision and intersection local times of independent Gaussian fields. Theorems 2.12 and 2.13 below generalize the results of Yan et al. (2009), Yan and Shen (2010) and Chen and Yan (2011) for fractional Brownian motion and related Gaussian processes.

\subsection{Smoothness of the collision local time}

Given $H=\left(H_{1}, \ldots, H_{N}\right) \in(0,1)^{N}$ and $K=\left(K_{1}, \ldots, K_{N}\right) \in(0,1)^{N}$, let $X^{H}=\left\{X^{H}(s), s \in \mathbb{R}^{N}\right\}$ and $X^{K}=\left\{X^{K}(t), t \in \mathbb{R}^{N}\right\}$ be two independent Gaussian random fields with values in $\mathbb{R}^{d}$ as defined in (1.1). We assume that the associate real-valued random fields $X_{0}^{H}$ and $X_{0}^{K}$ satisfy Conditions (C1) and $(\mathrm{C} 2)$ on interval $I \subseteq \mathbb{R}^{N}$ respectively with indices $H$ and with indices $K$.

The collision local time of $X^{H}$ and $X^{K}$ on $I$ is formally defined by

$$
L_{C}\left(X^{H}, X^{K}\right):=\int_{I} \delta\left(X^{H}(s)-X^{K}(s)\right) d s
$$

Theorem 2.12 Let $L_{C}\left(X^{H}, X^{K}\right)$ be the collision local time of $X^{H}$ and $X^{K}$ as above. Then

(i) $L_{C}\left(X^{H}, X^{K}\right) \in L^{2}(\Omega, \mathbb{P})$ if and only if $\sum_{j=1}^{N} \frac{1}{H_{j} \wedge K_{j}}>d$.

(ii) $L_{C}\left(X^{H}, X^{K}\right) \in D_{1}$ if and only if $\sum_{j=1}^{N} \frac{1}{H_{j} \wedge K_{j}}>d+2$.

Proof Consider the $(N, d)$ Gaussian field $Z=\{Z(t), t \in I\}$ defined by

$$
Z(t) \equiv X^{H}(t)-X^{K}(t), \quad \forall t \in I .
$$

Then the collision local time of $X^{H}$ and $X^{K}$ on $I$ is nothing but $L(0, I, Z)$, the local time of $Z$ on $I$ at $x=0$. Hence Theorem 2.12 follows from Theorems 2.6 and 2.10 once we verify that the real valued Gaussian field $Z_{0}(t)=X_{0}(t)-Y_{0}(t)$ satisfies (C1) and (C2) in the interval $I$ with indices $\left(H_{1} \wedge K_{1}, \ldots, H_{N} \wedge K_{N}\right) \in$ $(0,1)^{N}$. 
Since it is easy to show that $Z_{0}$ satisfies $(\mathrm{C} 1)$, we verify $(\mathrm{C} 2)$ only. By the definition of conditional variance and independence of $X^{H}$ and $X^{K}$, we have

$$
\begin{aligned}
& \operatorname{Var}\left(Z_{0}(t) \mid Z_{0}(s)\right)=\inf _{a \in \mathbb{R}}\left\{\mathbb{E}\left[\left(X_{0}^{H}(t)-a X_{0}^{H}(s)\right)^{2}+\left(X_{0}^{K}(t)-a X_{0}^{K}(s)\right)^{2}\right]\right\} \\
& \geqslant \inf _{a \in \mathbb{R}} \mathbb{E}\left[\left(X_{0}^{H}(t)-a X_{0}^{H}(s)\right)^{2}\right]+\inf _{b \in \mathbb{R}} \mathbb{E}\left[\left(X_{0}^{K}(t)-b X_{0}^{K}(s)\right)^{2}\right] \\
& =\operatorname{Var}\left(X_{0}^{H}(t) \mid X_{0}^{H}(s)\right)+\operatorname{Var}\left(X_{0}^{K}(t) \mid X_{0}^{K}(s)\right) \\
& \geqslant c\left(\sum_{j=1}^{N} \min \left\{\left|s_{j}-t_{j}\right|^{2 H_{j}}, t_{j}^{2 H_{j}}\right\}+\sum_{j=1}^{N} \min \left\{\left|s_{j}-t_{j}\right|^{2 K_{j}}, t_{j}^{2 K_{j}}\right\}\right) \\
& \geqslant c \sum_{j=1}^{N} \min \left\{\left|s_{j}-t_{j}\right|^{2\left(H_{j} \wedge K_{j}\right)}, t_{j}^{2\left(H_{j} \wedge K_{j}\right)}\right\}, \quad \forall s, t \in I,
\end{aligned}
$$

for some constant $c>0$. This verifies that $Z_{0}$ satisfies Condition $(\mathrm{C} 2)$.

\subsection{Smoothness of the intersection local time}

Let $H=\left(H_{1}, \ldots, H_{N_{1}}\right) \in(0,1)^{N_{1}}$ and $K=\left(K_{1}, \ldots, K_{N_{2}}\right) \in(0,1)^{N_{2}}$ be two constant vectors. Let $X^{H}=\left\{X^{H}(s), s \in \mathbb{R}^{N_{1}}\right\}$ and $X^{K}=\left\{X^{K}(t), t \in \mathbb{R}^{N_{2}}\right\}$ be two independent Gaussian random fields with values in $\mathbb{R}^{d}$ as defined in (1.1). We assume that the associate real-valued random fields $X_{0}^{H}$ and $X_{0}^{K}$ satisfy Conditions $(\mathrm{C} 1)$ and $(\mathrm{C} 2)$ respectively on interval $I_{1} \subseteq \mathbb{R}^{N_{1}}$ with indices $H=\left(H_{1}, \ldots, H_{N_{1}}\right)$ and on $I_{2} \subseteq \mathbb{R}^{N_{2}}$ with indices $K=\left(K_{1}, \ldots, K_{N_{2}}\right)$. Then the intersection local time of $X^{\bar{H}}$ and $X^{K}$ is formally defined by

$$
L_{I}\left(X^{H}, X^{K}\right):=\int_{I_{N_{1}} \times I_{N_{2}}} \delta\left(X^{H}(s)-X^{K}(t)\right) d s d t
$$

Theorem 2.13 Let $L_{I}\left(X^{H}, X^{K}\right)$ be the intersection local time of $X^{H}$ and $X^{K}$ as above. Then

(i) $L_{I}\left(X^{H}, X^{K}\right) \in L^{2}(\Omega, \mathbb{P})$ if and only if $\sum_{j=1}^{N_{1}} \frac{1}{H_{j}}+\sum_{j=1}^{N_{2}} \frac{1}{K_{j}}>d$.

(ii) $L_{I}\left(X^{H}, X^{K}\right) \in D_{1}$ if and only if $\sum_{j=1}^{N_{1}} \frac{1}{H_{j}}+\sum_{j=1}^{N_{2}} \frac{1}{K_{j}}>d+2$.

Proof Let $U=\left\{U(s, t),(s, t) \in I_{N_{1}} \times I_{N_{2}}\right\}$ be the $\left(N_{1}+N_{2}, d\right)$-Gaussian random field with mean 0 defined by

$$
U(s, t)=X^{H}(s)-X^{K}(t), \quad \forall s \in I_{N_{1}}, t \in I_{N_{2}} .
$$

Clearly, the intersection local time of $X^{H}$ and $X^{K}$ is nothing but $L\left(0, I_{N_{1}} \times\right.$ $\left.I_{N_{2}}, U\right)$, the local time of $U$ on $I_{N_{1}} \times I_{N_{2}}$ at $x=0$. One can verify that the Gaussian random field $U_{0}(s, t)=X_{0}^{H}(s)-X_{0}^{K}(t)$ satisfies Conditions $(\mathrm{C} 1)$ 
and $(\mathrm{C} 2)$ on the interval $I_{N_{1}} \times I_{N_{2}}$ with indices $\left(H_{1}, \ldots, H_{N_{1}}, K_{1}, \ldots, K_{N_{2}}\right) \in$ $(0,1)^{N_{1}+N_{2}}$. Therefore, the conclusions follow from Theorems 2.6] and 2.10.

\section{Self-intersection local times}

In this section, we study the existence and smoothness of self-intersection local times of an $(N, d)$-Gaussian random field $X=\left\{X(t), t \in \mathbb{R}^{N}\right\}$ as in

(1.1). These problems are more involved than the collision or intersection local times of independent Gaussian random fields, due to complexity of dependence structures of $X$. For earlier results for the Brownian sheet, fractional Brownian motion and related self-similar Gaussian processes, we refer to Imkeller and Weise (1995, 1999), Hu (2001), Jiang and Wang (2009). Their methods rely on special properties of the Brownian sheet or fractional Brownian motion. Our approach below is based on a weak form of local nondeterminism and is more general.

For any two compact intervals $I, J \subseteq \mathbb{R}^{N}$, the self-intersection local times of $X=\left\{X(t), t \in \mathbb{R}^{N}\right\}$ on $I$ and $J$ is formally defined by

$$
L_{S}(X, I \times J)=\int_{I \times J} \delta(X(s)-X(t)) d s d t .
$$

Define a $(2 N, d)$-Gaussian random field $V=\left\{V(s, t),(s, t) \in \mathbb{R}^{2 N}\right\}$ by

$$
V(s, t):=X(s)-X(t), s, t \in \mathbb{R}^{N} .
$$

Then the self-intersection local time of $X$ is $L(0, I \times J, V)$, the local time of $V$ on $I \times J$ at $x=0$.

Under the condition that $X_{0}$ satisfies Conditions $(\mathrm{C} 1)$ and $(\mathrm{C} 2)$ on both intervals $I$ and $J$, the Gaussian field $V_{0}(s, t)=X_{0}(s)-X_{0}(t)$ may not satisfy the corresponding $(\mathrm{C} 2)$ on $I \times J$. Therefore, we can not apply Theorems 2.6 and 2.10 directly. To overcome this difficulty, we will make use of the following condition:

(C3) There exists a positive constant $c_{12}$ such that for all $u, t^{1}, t^{2}, t^{3} \in[0,1]^{N}$,

$$
\operatorname{Var}\left(X_{0}(u) \mid X_{0}\left(t^{1}\right), X_{0}\left(t^{2}\right), X_{0}\left(t^{3}\right)\right) \geqslant c_{12} \sum_{j=1}^{N} \min _{0 \leqslant k \leqslant 3}\left|u_{j}-t_{j}^{k}\right|^{2 H_{j}},
$$

where $t_{j}^{0}=0, j=1,2, \ldots, N$.

Clearly, Condition (C2) is a special case of Condition (C3). It is known that multiparameter fractional Brownian motion and fractional Brownian sheets satisfy Conditions (C1) and (C3); see Pitt (1978) and Wu and Xiao (2007). More examples can be found in Xiao (2009). 
For two compact intervals $I, J \subseteq[0,1]^{N}$, we call them separated if

$$
\inf _{s \in I, t \in J}\left|s_{j}-t_{j}\right|>0 \text { for some } j=1,2, \ldots, N .
$$

Let $S \subseteq\{1, \ldots, N\}$ be the collection of all $j$ 's that satisfy (3.4) and let $S^{c}=$ $\{1, \ldots, N\} \backslash S$. Because $I$ and $J$ are compact, there exists $\varepsilon_{0}>0$ such that

$$
\inf _{s \in I, t \in J}\left|s_{j}-t_{j}\right| \geqslant \varepsilon_{0} \text { for } j \in S .
$$

We further call $I$ and $J$ partially separated if both $S$ and $S^{c}$ are nonempty, well separated if $S^{c}=\emptyset$, and not separated if $S=\emptyset$. Clearly, $I$ and $J$ are not separated iff $I \cap J \neq \emptyset$.

Similarly to Imkeller and Weisz (1999) for the Brownian sheet, we consider the self-intersection local times of $X$ on $I$ and $J$ by distinguishing three cases:

Case (i) $I, J \subseteq[0,1]^{N}$ are well separated.

Case (ii) $I, J \subseteq[0,1]^{N}$ are partially separated.

Case (iii) $I, J \subseteq[0,1]^{N}$ are not separated.

In Case (i), we have the following theorem.

Theorem 3.1 Let $X=\left\{X(t), t \in \mathbb{R}^{N}\right\}$ be an $(N, d)$-Gaussian random field defined by (1.1) with $X_{0}$ satisfying Conditions (C1) and (C3) and let $L_{S}(X, I \times J)$ be the self-intersection local time of $X$ on $I$ and $J$. If I and $J$ are well separated, then the following statements hold:

(i) $L_{S}(X, I \times J) \in L^{2}(\Omega, \mathbb{P})$ if and only if $2 \sum_{j=1}^{N} \frac{1}{H_{j}}>d$.

(ii) $L_{S}(X, I \times J) \in D_{1}$ if and only if $2 \sum_{j=1}^{N} \frac{1}{H_{j}}>d+2$.

Proof Since the Gaussian field $X_{0}$ satisfies $(\mathrm{C} 1)$ on $I$ and $J$, we see that for any $(s, t),\left(s^{\prime}, t^{\prime}\right) \in I \times J$,

$$
\mathbb{E}\left[\left(V_{0}(s, t)-V_{0}\left(s^{\prime}, t^{\prime}\right)\right)^{2}\right] \leqslant c\left[\sum_{j=1}^{N}\left|s_{j}-s_{j}^{\prime}\right|^{2 H_{j}}+\sum_{j=1}^{N}\left|t_{j}-t_{j}^{\prime}\right|^{2 H_{j}}\right] .
$$

Thus the Gaussian field $V_{0}(s, t)=X_{0}^{H}(s)-X_{0}^{H}(t)$ satisfies $(\mathrm{C} 1)$ on $I \times J$ with indices $\left(H_{1}, \ldots, H_{N}, H_{1}, \ldots, H_{N}\right) \in(0,1)^{2 N}$. To verify that $V_{0}$ also satisfies (C2), we see that (C3) implies

$$
\begin{aligned}
\operatorname{Var}\left(V_{0}(s, t) \mid V_{0}\left(s^{\prime}, t^{\prime}\right)\right) & \geqslant \operatorname{Var}\left(X_{0}(t) \mid X_{0}(s), X_{0}\left(s^{\prime}\right), X_{0}\left(t^{\prime}\right)\right) \\
& \geqslant c_{12} \sum_{j=1}^{N} \min \left\{\left|t_{j}-s_{j}\right|^{2 H_{j}},\left|t_{j}-s_{j}^{\prime}\right|^{2 H_{j}},\left|t_{j}-t_{j}^{\prime}\right|^{2 H_{j}}, t_{j}^{2 H_{j}}\right\} \\
& \geqslant c_{13} \sum_{j=1}^{N} \min \left\{\left|t_{j}-t_{j}^{\prime}\right|^{2 H_{j}}, t_{j}^{2 H_{j}}\right\}
\end{aligned}
$$


thanks to the fact that $\left|t_{j}-s_{j}\right| \geqslant \varepsilon_{0}$ and $\left|t_{j}-s_{j}^{\prime}\right| \geqslant \varepsilon_{0}$. Here the constant $c_{13}$ depends on $\varepsilon_{0}$. By the same token, we have

$$
\operatorname{Var}\left(V_{0}(s, t) \mid V_{0}\left(s^{\prime}, t^{\prime}\right)\right) \geqslant c_{13} \sum_{j=1}^{N} \min \left\{\left|s_{j}-s_{j}^{\prime}\right|^{2 H_{j}}, s_{j}^{2 H_{j}}\right\} .
$$

Adding up these two inequalities shows

$$
\begin{aligned}
& \operatorname{Var}\left(V_{0}(s, t) \mid V_{0}\left(s^{\prime}, t^{\prime}\right)\right) \\
& \geqslant \frac{c_{13}}{2}\left[\sum_{j=1}^{N} \min \left\{\left|s_{j}-s_{j}^{\prime}\right|^{2 H_{j}}, s_{j}^{2 H_{j}}\right\}+\sum_{j=1}^{N} \min \left\{\left|t_{j}-t_{j}^{\prime}\right|^{2 H_{j}}, t_{j}^{2 H_{j}}\right\}\right] .
\end{aligned}
$$

This proves that $V_{0}$ satisfies $(\mathrm{C} 2)$ on $I \times J$ with $\left(H_{1}, \ldots, H_{N}, H_{1}, \ldots, H_{N}\right)$ $\in(0,1)^{2 N}$. Therefore, the conclusions follow from Theorems 2.6] and 2.10,

Now we consider Case (ii), e.g. the two compact intervals $I$ and $J$ are partially separated. In this case, both $S$ and $S^{c}$ are nonempty sets. For concreteness, we may assume that $I=[a, a+\langle h\rangle], J=[b, b+\langle h\rangle]$, where $b_{j}>a_{j}+h$ for $j \in S$ and $a_{j}=b_{j}$ for $j \in S^{c}$. Then (3.5) holds with $\varepsilon_{0}=\min \left\{b_{j}-a_{j}-h, j \in S\right\}$. Note that, when $X$ is the $(N, d)$ Brownian sheet, the existence condition in (i) in the following theorem coincides with that in Theorem 3 of Imkeller and Weisz (1995, 1999).

Theorem 3.2 Let $X=\left\{X(t), t \in \mathbb{R}^{N}\right\}$ be an $(N, d)$-Gaussian random field as in Theorem 3.1. Let $I$ and $J$ be partially separated as described above. Then the following statements hold:

(i) $L_{S}(X, I \times J) \in L^{2}(\Omega, \mathbb{P})$ if $2 \sum_{j \in S} \frac{1}{H_{j}}+\sum_{j \in S^{c}} \frac{1}{H_{j}}>d$.

(ii) $L_{S}(X, I \times J) \notin L^{2}(\Omega, \mathbb{P})$ if $2 \sum_{j=1}^{N} \frac{1}{H_{j}} \leqslant d$.

(iii) $L_{S}(X, I \times J) \in D_{1}$ if $2 \sum_{j \in S} \frac{1}{H_{j}}+\sum_{j \in S^{c}} \frac{1}{H_{j}}>d+2$.

(iv) $L_{S}(X, I \times J) \notin D_{1}$ if $2 \sum_{j=1}^{N} \frac{1}{H_{j}} \leqslant d+2$.

Proof We prove Part (i) at first. By Lemma 2.5, we only need to prove that if $2 \sum_{j \in S} \frac{1}{H_{j}}+\sum_{j \in S^{c}} \frac{1}{H_{j}}>d$ then

$$
\mathscr{J}:=\int_{(I \times J)^{2}} \frac{d s d t d s^{\prime} d t^{\prime}}{\left[\operatorname{det} \operatorname{Cov}\left(V_{0}(s, t), V_{0}\left(s^{\prime}, t^{\prime}\right)\right)\right]^{d / 2}}<\infty .
$$

By the definition of conditional variance and (C3), we see that for any $(s, t),\left(s^{\prime}, t^{\prime}\right) \in$ 
$I \times J$,

$$
\begin{aligned}
\operatorname{Var}( & \left.V_{0}(s, t) \mid V_{0}\left(s^{\prime}, t^{\prime}\right)\right) \geqslant \operatorname{Var}\left(X_{0}(t) \mid X_{0}(s), X_{0}\left(s^{\prime}\right), X_{0}\left(t^{\prime}\right)\right) \\
\geqslant & c_{12} \sum_{j=1}^{N} \min \left\{\left|t_{j}-s_{j}\right|^{2 H_{j}},\left|t_{j}-s_{j}^{\prime}\right|^{2 H_{j}},\left|t_{j}-t_{j}^{\prime}\right|^{2 H_{j}}, t_{j}^{2 H_{j}}\right\} \\
\geqslant & c_{14}\left(\sum_{j \in S} \min \left\{\left|t_{j}-t_{j}^{\prime}\right|^{2 H_{j}}, t_{j}^{2 H_{j}}\right\}\right. \\
& \left.+\sum_{j \in S^{c}} \min \left\{\left|t_{j}-s_{j}\right|^{2 H_{j}},\left|t_{j}-s_{j}^{\prime}\right|^{2 H_{j}},\left|t_{j}-t_{j}^{\prime}\right|^{2 H_{j}}, t_{j}^{2 H_{j}}\right\}\right),
\end{aligned}
$$

thanks to the fact that if $j \in S$, then $\left|t_{j}-s_{j}\right| \geqslant \varepsilon_{0}$ and $\left|t_{j}-s_{j}^{\prime}\right| \geqslant \varepsilon_{0}$. By the same token, we have

$$
\begin{aligned}
& \operatorname{Var}\left(V_{0}(s, t) \mid V_{0}\left(s^{\prime}, t^{\prime}\right)\right) \geqslant c_{14}\left(\sum_{j \in S} \min \left\{\left|s_{j}-s_{j}^{\prime}\right|^{2 H_{j}}, s_{j}^{2 H_{j}}\right\}\right. \\
& \left.+\sum_{j \in S^{c}} \min \left\{\left|s_{j}-t_{j}\right|^{2 H_{j}},\left|s_{j}-s_{j}^{\prime}\right|^{2 H_{j}},\left|s_{j}-t_{j}^{\prime}\right|^{2 H_{j}}, s_{j}^{2 H_{j}}\right\}\right) .
\end{aligned}
$$

Combining (3.8) and (3.9), we have

$$
\begin{aligned}
& \operatorname{Var}\left(V_{0}(s, t) \mid V_{0}\left(s^{\prime}, t^{\prime}\right)\right) \\
& \geqslant c_{14}\left[\sum_{j \in S}\left(\min \left\{\left|t_{j}-t_{j}^{\prime}\right|^{2 H_{j}}, t_{j}^{2 H_{j}}\right\}+\min \left\{\left|s_{j}-s_{j}^{\prime}\right|^{2 H_{j}}, s_{j}^{2 H_{j}}\right\}\right)\right. \\
& \left.\quad+\sum_{j \in S^{c}} \min \left\{\left|s_{j}-t_{j}\right|^{2 H_{j}},\left|s_{j}-s_{j}^{\prime}\right|^{2 H_{j}},\left|s_{j}-t_{j}^{\prime}\right|^{2 H_{j}}, s_{j}^{2 H_{j}}\right\}\right] .
\end{aligned}
$$

Note that, in (3.10), only one sum over $S^{c}$ in (3.8) and (3.9) is kept. This is due to the fact that, when integrating $d s_{j}$ for $j \in S^{c}$, all the other variables, $s_{j}^{\prime}, t_{j}$ and $t_{j}^{\prime}$, will disappear [see Lemma 2.2]. This situation is different from the case when we integrate $d s_{j}$ for $j \in S$.

Since $I$ and $J$ are partially separated (i.e., $S \neq \emptyset$ ), we have

$$
\operatorname{Var}\left(V_{0}\left(s^{\prime}, t^{\prime}\right)\right)=\mathbb{E}\left[\left(X_{0}\left(s^{\prime}\right)-X_{0}\left(t^{\prime}\right)\right)^{2}\right] \asymp 1, \quad \forall s^{\prime} \in I, t^{\prime} \in J .
$$

It follows from (3.10) and (3.11) that the integral $\mathscr{J}$ in (3.7) is at most

$$
\begin{aligned}
& \int_{(I \times J)^{2}}\left[\sum_{j \in S}\left(\min \left\{\left|t_{j}-t_{j}^{\prime}\right|^{H_{j}}, t_{j}^{H_{j}}\right\}+\min \left\{\left|s_{j}-s_{j}^{\prime}\right|^{H_{j}}, s_{j}^{H_{j}}\right\}\right)\right. \\
& \left.+\sum_{j \in S^{c}} \min \left\{\left|s_{j}-t_{j}\right|^{H_{j}},\left|s_{j}-s_{j}^{\prime}\right|^{H_{j}},\left|s_{j}-t_{j}^{\prime}\right|^{H_{j}}, s_{j}^{H_{j}}\right\}\right]^{-d} d s d t d s^{\prime} d t^{\prime} .
\end{aligned}
$$


Similarly to the argument in the proofs of (2.12) and (2.21), we integrate iteratively and apply Lemmas 2.1-2.3 to show that the integral in (3.12) is finite if $2 \sum_{j \in S=1} \frac{1}{H_{j}}+\sum_{j \in S^{c}} \frac{1}{H_{j}}>d$. This proves the sufficiency in Part (i).

Next we prove Part (ii). For any $(s, t),\left(s^{\prime}, t^{\prime}\right) \in I \times J$, Condition (C1) implies that

$$
\begin{aligned}
\operatorname{Var}\left(V_{0}(s, t) \mid V_{0}\left(s^{\prime}, t^{\prime}\right)\right) & \leqslant \mathbb{E}\left[\left(X_{0}(s)-X_{0}(t)-X_{0}\left(s^{\prime}\right)+X_{0}\left(t^{\prime}\right)\right)^{2}\right] \\
& \leqslant c \sum_{j=1}^{N}\left(\left|s_{j}-s_{j}^{\prime}\right|^{2 H_{j}}+\left|t_{j}-t_{j}^{\prime}\right|^{2 H_{j}}\right) .
\end{aligned}
$$

It follows from (3.11) and (3.13) that

$$
\operatorname{det} \operatorname{Cov}\left(V_{0}(s, t), V_{0}\left(s^{\prime}, t^{\prime}\right)\right) \leqslant c \sum_{j=1}^{N}\left(\left|s_{j}-s_{j}^{\prime}\right|^{2 H_{j}}+\left|t_{j}-t_{j}^{\prime}\right|^{2 H_{j}}\right) .
$$

This implies, by using Lemma 2.1 repeatedly, that the integral $\mathscr{J}$ in (3.7) is infinite if $2 \sum_{j=1}^{N} \frac{1}{H_{j}} \leqslant d$.

In order to prove Part (iii), by Lemma 2.9, it suffices to show that, if $2 \sum_{j \in S} \frac{1}{H_{j}}+\sum_{j \in S^{c}} \frac{1}{H_{j}}>d+2$, then

$$
\mathscr{K}=\int_{(I \times J)^{2}} \frac{\left[\mathbb{E}\left(V_{0}(s, t) V_{0}\left(s^{\prime}, t^{\prime}\right)\right)\right]^{2}}{\left[\operatorname{det} \operatorname{Cov}\left(V_{0}(s, t), V_{0}\left(s^{\prime}, t^{\prime}\right)\right)\right]^{\frac{d+2}{2}}} d s d t d s^{\prime} d t^{\prime}<\infty .
$$

For any $(s, t),\left(s^{\prime}, t^{\prime}\right) \in I \times J$, we use the Cauchy-Schwarz inequality and (C1) again to show that

$$
\left[\mathbb{E}\left(V_{0}(s, t) V_{0}\left(s^{\prime}, t^{\prime}\right)\right)\right]^{2} \leqslant \mathbb{E}\left[V_{0}^{2}(s, t)\right] \mathbb{E}\left[V_{0}^{2}\left(s^{\prime}, t^{\prime}\right)\right] \leqslant c .
$$

Similar to the argument in (3.11) and (3.12), we derive from (3.16) that the integral $\mathscr{K}$ in (3.15) is, up to a constant, bounded from above by

$$
\begin{aligned}
& \int_{(I \times J)^{2}}\left[\sum_{j \in S}\left(\min \left\{\left|t_{j}-t_{j}^{\prime}\right|^{H_{j}}, t_{j}^{H_{j}}\right\}+\min \left\{\left|s_{j}-s_{j}^{\prime}\right|^{H_{j}}, s_{j}^{H_{j}}\right\}\right)\right. \\
& \left.+\sum_{j \in S^{c}} \min \left\{\left|s_{j}-s_{j}\right|^{H_{j}},\left|s_{j}-s_{j}^{\prime}\right|^{H_{j}},\left|s_{j}-t_{j}^{\prime}\right|^{H_{j}}, s_{j}^{H_{j}}\right\}\right]^{-(d+2)} d s d t d s^{\prime} d t^{\prime} .
\end{aligned}
$$

Again exactly like what we did in the proof of (2.12), we can show that the integral in (3.17) is finite provided $2 \sum_{j \in S} 1 / H_{j}+\sum_{j \in S^{c}} 1 / H_{j}>d+2$. This proves Part (iii).

Since the function $\mathbb{E}\left(V_{0}(s, t) V_{0}\left(s^{\prime}, t^{\prime}\right)\right)$ is uniform continuous for $\left(s, t, s^{\prime}, t^{\prime}\right) \in$ $(I \times J)^{2}$ and (3.11) holds, there exist positive constants $\delta$ and $c$ such that $\mathbb{E}\left(V_{0}(s, t) V_{0}\left(s^{\prime}, t^{\prime}\right)\right) \geqslant c$ for all $\left(s, t, s^{\prime}, t^{\prime}\right) \in(I \times J)^{2}$ such that $\left|(s, t)-\left(s^{\prime}, t^{\prime}\right)\right| \leqslant$ 
$\delta$. Hence the proof of Part (iv) is quite similar to the proof of Part (ii). We leave the details to the interested reader.

Part (ii) and (iv) in Theorem 3.2 can be improved if we have more information on the dependence structure of $V_{0}(s, t)=X_{0}(s)-X_{0}(t)$, as shown by the following theorem.

Theorem 3.3 If, in addition to the assumptions of Theorem 3.2, $X_{0}$ satisfies the following condition:

(C4) There exists a positive constant $c_{15}$ such that for all $(s, t),\left(s^{\prime}, t^{\prime}\right) \in I \times J$,

$$
\begin{aligned}
& \operatorname{Var}\left(X_{0}(s)-X_{0}(t) \mid X_{0}\left(s^{\prime}\right)-X_{0}\left(t^{\prime}\right)\right) \\
& \quad \leqslant c_{15}\left(\sum_{j \in S}\left(\left|t_{j}-t_{j}^{\prime}\right|^{2 H_{j}}+\left|s_{j}-s_{j}^{\prime}\right|^{2 H_{j}}\right)+\sum_{j \in S^{c}}\left|s_{j}-t_{j}\right|^{2 H_{j}}\right) .
\end{aligned}
$$

Then the following statements hold:

(i) $L_{S}\left(X^{H}, I \times J\right) \in L^{2}(\Omega, \mathbb{P})$ if and only if $2 \sum_{j \in S} \frac{1}{H_{j}}+\sum_{j \in S^{c}} \frac{1}{H_{j}}>d$.

(ii) $L_{S}\left(X^{H}, I \times J\right) \in D_{1}$ if and only if $2 \sum_{j \in S} \frac{1}{H_{j}}+\sum_{j \in S^{c}} \frac{1}{H_{j}}>d+2$.

Remark 3.4 Observe that Condition (C4) is automatically satisfied if $S=\emptyset$. If $X_{0}=\left\{X_{0}(t), t \in \mathbb{R}^{N}\right\}$ is an "additive fractional Brownian motion" defined by

$$
X_{0}(t)=B^{H_{1}}\left(t_{1}\right)+\cdots+B^{H_{N}}\left(t_{N}\right), \quad \forall t \in \mathbb{R}^{N},
$$

where $B^{H_{1}}, \ldots, B^{H_{N}}$ are independent fractional Brownian motions with indices $H_{1}, \ldots, H_{N}$, respectively. Then it is easy to see that Condition (C4) is satisfied. When $X_{0}$ is the Brownian sheet, then by using the independence of increments over intervals, one can check that (C4) also holds.

Proof of Theorem 3.3 Sufficiencies of the condition in (i) and (ii) have been proved in Theorem 3.2. Note that (C4) and (3.11) imply

$$
\mathscr{J} \geqslant \int_{(I \times J)^{2}} \frac{d s d t d s^{\prime} d t^{\prime}}{\left[\sum_{j \in S}\left(\left|s_{j}-s_{j}^{\prime}\right|^{H_{j}}+\left|t_{j}-t_{j}^{\prime}\right|^{H_{j}}\right)+\sum_{j \in S^{c}}\left|s_{j}-t_{j}\right|^{H_{j}}\right]^{d}} .
$$

By applying Lemma 2.1, it can be verified that the last integral diverges when $2 \sum_{j \in S} \frac{1}{H_{j}}+\sum_{j \in S^{c}} \frac{1}{H_{j}} \leqslant d$. This proves the necessity in (i). The proof of necessity in (ii) is similar and is omitted.

Finally, we consider Case (iii), e.g. the two compact intervals $I$ and $J$ are not separated in any direction. So $S=\emptyset$. Compared with Case (ii), we note that, on one hand, (3.11) fails and, on the other hand, Condition (C4) holds automatically. For concreteness, we assume that $I=J=[0,1]^{N}$. 
Theorem 3.5 Let $X=\left\{X(t), t \in \mathbb{R}^{N}\right\}$ be an $(N, d)$-Gaussian random field as in Theorem [3.1. Then the following statements hold:

(i) $L_{S}(X, I \times I) \in L^{2}(\Omega, \mathbb{P})$ if and only if $\sum_{j=1}^{N} \frac{1}{H_{j}}>d$.

(ii) $L_{S}(X, I \times I) \in D_{1}$ if $\sum_{j=1}^{N} \frac{1}{H_{j}}>d+2$.

(iii) $L_{S}\left(X^{H}, I \times I\right) \notin D_{1}$ if

$$
\sum_{j=1}^{N} \frac{1}{H_{j}} \leqslant \max \left\{\frac{d+2}{2}, \frac{2 d}{3}\right\} .
$$

Before proving this theorem, we compare its conditions with the results in Imkeller and Weisz $(1995,1999)$ and $\mathrm{Hu}$ (2001).

Remark 3.6 (a) When $X$ is the $(N, d)$ Brownian sheet, then our existence condition in (i) coincides with that in Theorem 1 of Imkeller and Weisz (1995, 1999). When $X$ is a fractional Brownian motion $B^{H}=\left\{B^{H}(t), t \in\right.$ $\mathbb{R}\}$, our condition in (ii) becomes $H(d+2)<1$, which is stronger than that in $\mathrm{Hu}$ (2001, Theorem 3.2).

(b) Little has been known about optimal necessary condition for $L_{S}(X, I \times I) \in$ $D_{1}$ for a Gaussian random field $X$. Our condition (3.20) is the first general result of this kind. When $X$ is a fractional Brownian motion $B^{H}$, (3.20) becomes $H \geqslant \min \left\{\frac{3}{2 d}, \frac{2}{d+2}\right\}$, which is the complement of the sufficient condition of $H u$ (2001, Theorem 3.2). Hence, we have proven that, for fractional Brownian motion $B^{H}=\left\{B^{H}(t), t \in \mathbb{R}\right\}$ in $\mathbb{R}^{d}$ and $I=[0,1]$, $L_{S}\left(B^{H}, I \times I\right) \in D_{1}$ if and only if $H<\min \left\{\frac{3}{2 d}, \frac{2}{d+2}\right\}$.

Proof of Theorem 3.5 We prove Parts (i) at first. Notice that by Lemma 2.5. we only need to prove that

$$
\mathscr{J}=\int_{I^{4}} \frac{d s d t d s^{\prime} d t^{\prime}}{\left[\operatorname{det} \operatorname{Cov}\left(V_{0}(s, t), V_{0}\left(s^{\prime}, t^{\prime}\right)\right)\right]^{d / 2}}<\infty
$$

if and only if $\sum_{j=1}^{N} \frac{1}{H_{j}}>d$. For any $(s, t),\left(s^{\prime}, t^{\prime}\right) \in I \times I$, we use Condition (C1) to obtain that

$$
\begin{aligned}
\operatorname{det} \operatorname{Cov}\left(V_{0}(s, t), V_{0}\left(s^{\prime}, t^{\prime}\right)\right) & \leqslant \mathbb{E}\left(V_{0}^{2}(s, t)\right) \mathbb{E}\left(V_{0}^{2}\left(s^{\prime}, t^{\prime}\right)\right) \\
& \leqslant c\left(\sum_{j=1}^{N}\left|s_{j}-t_{j}\right|^{2 H_{j}}\right)\left(\sum_{j=1}^{N}\left|s_{j}^{\prime}-t_{j}^{\prime}\right|^{2 H_{j}}\right) .
\end{aligned}
$$

This, together with (3.21), implies

$$
\begin{aligned}
\mathscr{J} & \geqslant c \int_{I^{2}} \frac{d s d t}{\left(\sum_{j=1}^{N}\left|s_{j}-t_{j}\right|^{2 H_{j}}\right)^{d / 2}} \int_{I^{2}} \frac{d s^{\prime} d t^{\prime}}{\left(\sum_{j=1}^{N}\left|s_{j}^{\prime}-t_{j}^{\prime}\right|^{2 H_{j}}\right)^{d / 2}} \\
& \geqslant c\left(\int_{I} \frac{d t}{\left(\sum_{\ell=1}^{N} t_{\ell}^{H_{\ell}}\right)^{d}}\right)^{2} .
\end{aligned}
$$


By using Lemma 2.1, it is elementary to verify that the last integral in (3.23) is infinite provided $\sum_{j=1}^{N} 1 / H_{j} \leqslant d$. Hence, we prove the necessity of Part (i).

To prove the sufficiency in Parts (i), we apply Condition (C3) to see that for any $(s, t),\left(s^{\prime}, t^{\prime}\right) \in I \times I$,

$$
\begin{aligned}
\operatorname{Var}\left(V_{0}(s, t) \mid V_{0}\left(s^{\prime}, t^{\prime}\right)\right) & \geqslant \operatorname{Var}\left(X_{0}(t) \mid X_{0}(s), X_{0}\left(s^{\prime}\right), X_{0}\left(t^{\prime}\right)\right) \\
& \geqslant c \sum_{j=1}^{N} \min \left\{\left|t_{j}-s_{j}\right|^{2 H_{j}},\left|t_{j}-s_{j}^{\prime}\right|^{2 H_{j}},\left|t_{j}-t_{j}^{\prime}\right|^{2 H_{j}}, t_{j}^{2 H_{j}}\right\} .
\end{aligned}
$$

Moreover, we also have

$$
\operatorname{Var}\left(V_{0}\left(s^{\prime}, t^{\prime}\right)\right) \geqslant \operatorname{Var}\left(X_{0}\left(t^{\prime}\right) \mid X_{0}\left(s^{\prime}\right)\right) \geqslant c \sum_{j=1}^{N} \min \left\{\left|t_{j}^{\prime}-s_{j}^{\prime}\right|^{2 H_{j}}, t_{j}^{\prime 2 H_{j}}\right\} .
$$

Combining the above two inequalities with (3.21) yields

$$
\begin{gathered}
\mathscr{J} \leqslant c \int_{I^{4}}\left[\sum_{j=1}^{N} \min \left\{\left|t_{j}-s_{j}\right|^{H_{j}},\left|t_{j}-s_{j}^{\prime}\right|^{H_{j}},\left|t_{j}-t_{j}^{\prime}\right|^{H_{j}}, t_{j}^{H_{j}}\right\}\right]^{-d} \\
\times\left[\sum_{j=1}^{N} \min \left\{\left|t_{j}^{\prime}-s_{j}^{\prime}\right|^{H_{j}}, t_{j}^{\prime H_{j}}\right\}\right]^{-d} d s d t d s^{\prime} d t^{\prime}
\end{gathered}
$$

Similarly to the proof of (2.12), we integrate $d t_{1}, \ldots, d t_{N}, d t_{1}^{\prime}, \ldots, d t_{N}^{\prime}$ to show that the integral in (3.24) is finite provided $\sum_{\ell=1}^{N} 1 / H_{\ell}>d$. This proves the sufficiency of Part (i).

In order to prove Part (ii), by Lemma 2.9, it suffices to verify that if $\sum_{j=1}^{N} \frac{1}{H_{j}}>d+2$, then

$$
\mathscr{K}=\int_{I^{4}} \frac{\left[\mathbb{E}\left(V_{0}(s, t) V_{0}\left(s^{\prime}, t^{\prime}\right)\right)\right]^{2}}{\left[\operatorname{det} \operatorname{Cov}\left(V_{0}(s, t), V_{0}\left(s^{\prime}, t^{\prime}\right)\right)\right]^{\frac{d+2}{2}}} d s d t d s^{\prime} d t^{\prime}<\infty .
$$

For any $(s, t),\left(s^{\prime}, t^{\prime}\right) \in I \times I$, the Cauchy-Schwarz inequality and (C1) imply

$$
\left[\mathbb{E}\left(V_{0}(s, t) V_{0}\left(s^{\prime}, t^{\prime}\right)\right)\right]^{2} \leqslant \mathbb{E}\left[V_{0}^{2}(s, t)\right] \mathbb{E}\left[V_{0}^{2}\left(s^{\prime}, t^{\prime}\right)\right] \leqslant c .
$$

Similar to the argument in Part (i), we derive from (3.26) that

$$
\begin{aligned}
\mathscr{J} \leqslant c \int_{I^{4}}\left[\sum_{j=1}^{N} \min \left\{\left|t_{j}-s_{j}\right|^{H_{j}},\left|t_{j}-s_{j}^{\prime}\right|^{H_{j}},\left|t_{j}-t_{j}^{\prime}\right|^{H_{j}}, t_{j}^{H_{j}}\right\}\right]^{-(d+2)} \\
\times\left[\sum_{j=1}^{N} \min \left\{\left|t_{j}^{\prime}-s_{j}^{\prime}\right|^{H_{j}},{t^{\prime}}_{j}^{H_{j}}\right\}\right]^{-(d+2)} d s d t d s^{\prime} d t^{\prime} .
\end{aligned}
$$


Again we integrate in the order of $d t_{1}, \ldots, d t_{N}, d t_{1}^{\prime}, \ldots, d t_{N}^{\prime}$ to show that the integral in (3.27) is finite provided $\sum_{\ell=1}^{N} 1 / H_{\ell}>d+2$. This proves (3.25) and hence Part (ii).

Finally we prove Part (iii). By taking two disjoint sub-intervals and argue as in the proof of Theorem 3.1, one can see easily that if $2 \sum_{j=1}^{N} \frac{1}{H_{j}} \leqslant d+2$, then the integral $\mathscr{K}$ in (3.25) diverges and, consequently, $L_{S}\left(X^{H}, I \times I\right) \notin D_{1}$.

It remains to show that the integral $\mathscr{K}$ also diverges if $3 \sum_{j=1}^{N} \frac{1}{H_{j}} \leqslant 2 d$. To this end, we write $\rho(s, t)=\sqrt{\mathbb{E}\left(V_{0}(s, t)^{2}\right)}$. It will be useful to note that $\rho(s, t)$ is a pseudo-metric on $\mathbb{R}^{2 N}$. Since

$$
\left.\mathbb{E}\left(V_{0}(s, t)-V_{0}\left(s^{\prime}, t^{\prime}\right)\right)^{2}\right) \leqslant 2\left(\rho(s, t)^{2}+\rho\left(s^{\prime}, t^{\prime}\right)^{2}\right),
$$

we see that, if $\rho\left(s, s^{\prime}\right) \leqslant \frac{1}{2} \rho(s, t)$ and $\rho\left(t, t^{\prime}\right) \leqslant \frac{1}{2} \rho(s, t)$, then

$$
\begin{aligned}
\mathbb{E}\left(V_{0}(s, t) V_{0}\left(s^{\prime}, t^{\prime}\right)\right)= & \frac{1}{2}\left[\mathbb{E}\left(V_{0}(s, t)^{2}\right)+\mathbb{E}\left(V_{0}\left(s^{\prime}, t^{\prime}\right)^{2}\right)\right. \\
& \left.\quad-\mathbb{E}\left(\left(V_{0}(s, t)-V_{0}\left(s^{\prime}, t^{\prime}\right)\right)^{2}\right)\right] \\
\geqslant & \frac{1}{2} \mathbb{E}\left(V_{0}\left(s^{\prime}, t^{\prime}\right)^{2}\right) .
\end{aligned}
$$

Let $B_{\rho}(s, t)=\left\{\left(s^{\prime}, t^{\prime}\right) \in I^{2}: \rho\left(s, s^{\prime}\right) \leqslant \frac{1}{2} \rho(s, t), \rho\left(t, t^{\prime}\right) \leqslant \frac{1}{2} \rho(s, t)\right\}$. It follows from (3.25), (3.22) and (3.28) that

$$
\mathscr{K} \geqslant c \int_{I^{2}} \frac{d s d t}{\rho(s, t)^{d+2}} \int_{B_{\rho}(s, t)} \frac{d s^{\prime} d t^{\prime}}{\rho\left(s^{\prime}, t^{\prime}\right)^{d-2}} \geqslant \int_{I^{2}} \frac{d s d t}{\rho(s, t)^{2(d-Q)}},
$$

where $Q=\sum_{j=1}^{N} \frac{1}{H_{j}}$. In obtaining the last inequality, we have used the fact that $\rho\left(s^{\prime}, t^{\prime}\right) \leqslant 2 \rho(s, t)$ for all $\left(s^{\prime}, t^{\prime}\right) \in B_{\rho}(s, t)$, and the Lebesgue measure of $B_{\rho}(s, t)$ is $c \rho(s, t)^{2 Q}$. Under Conditions $(\mathrm{C} 1), \rho(s, t) \leqslant c_{1} \sum_{j=1}^{N}\left|s_{j}-t_{j}\right|^{H_{j}}$ for all $s, t \in I^{N}$. We can apply Lemma 2.1 to show that the last integral in (3.29) diverges if and only if $Q \leqslant 2(d-Q)$. This proves $\mathscr{K}=\infty$ when $3 Q \leqslant 2 d$. The proof of Theorem 3.5 is finished.

The following are concluding remarks.

Remark 3.7 (a) It is known that Conditions (C1) and (C3) are satisfied by a large class of Gaussian random fields including $N$-parameter fractional Brownian motion [Pitt (1978)], fractional Brownian sheets [Wu and Xiao (2007), Xiao (2009)] and stochastic heat equation driver by spacetime Gaussian noises [Dalang, et al. (2015), Tudor and Xiao (2015)]. Hence Theorems 3.1 and 3.2 can be applied directly to these Gaussian random fields. However, despite the conditions given by Theorems 3.3 and 3.5, the problem for finding necessary and sufficient conditions for $L_{S}(X, I \times I) \in D_{1}$ is still open for a general Gaussian random field. It would be interesting to solve this problem. 
(b) Another interesting question is to remove the i.i.d. assumption on the coordinate random fields $X_{1}, \ldots X_{d}$ in (1.1). While the results of this paper can be extended to Gaussian random fields with independent, but non-identically distributed components, it seems more difficult to remove the independence assumption. Some preliminary results have been proved by Eddahbi, et al. (2005, 2007) for vector-valued fractional Brownian sheets, but their conditions may not be optimal.

Acknowledgements Research of Z. Chen and D. Wu was partially supported by the National Natural Science Foundation of China (Grant No. 11371321). Research of Y. Xiao was partially supported by the NSF Grants DMS-1307470 and DMS-1309856.

\section{References}

1. Ayache A., Wu D. and Xiao Y. Joint continuity of the local times of fractional Brownian sheets. Ann Inst H Poincaré Probab Statist, 2008, 44: 727-748

2. Ayache A. and Xiao Y. Asymptotic properties and Hausdorff dimensions of fractional Brownian sheets. J Fourier Anal Appl, 2005, 11: 407-439

3. Biermé H., Lacaux C. and Xiao Y. Hitting probabilities and the Hausdorff dimension of the inverse images of anisotropic Gaussian random fields. Bull London Math Soc, 2009, 41: 253-273

4. Chen C. and Yan L. Remarks on the intersection local time of fractional Brownian motions. Statist Probab Lett, 2011, 81: 1003-1012

5. Chen Z. and Xiao Y. On intersections of independent anisotropic Gaussian random fields. Sci China Math, 2012, 55: 2217-2232

6. Dalang R. C., Mueller C. and Xiao Y. Polarity of points for a wide class of Gaussian random fields. Submitted, 2015

7. Eddahbi, M., Lacayo, R., Solé, J. L., Vives, J. and Tudor, C. A. Regularity of the local time for the $d$-dimensional fractional Brownian motion with $N$-parameters. Stoch Anal Appl, 2005, 23: 383-400

8. Eddahbi, M., Lacayo, R., Solé, J. L., Vives, J. and Tudor, C. A. Renormalization of the local time for the $d$-dimensional fractional Brownian motion with $N$ parameters. Nagoya Math J, 2007, 186: 173-191

9. Eddahbi, M. and Vives, J. Chaotic expansion and smoothness of some functionals of the fractional Brownian motion. J Math Kyoto Univ, 2003, 43: 349-368

10. Geman, D. and Horowitz, J. Occupation densities. Ann Probab, 1980, 8: 1-67

11. $\mathrm{Hu}$ Y. Self-intersection local time of fractional Brownian motion-via chaos expansion. J Math Kyoto Univ, 2001, 41: 233-250

12. $\mathrm{Hu} \mathrm{Y}$. and $\varnothing \mathrm{ksendal}, \mathrm{B}$. Chaos expansion of local time of fractional Brownian motions. Stoch Anal Appl, 2002, 20: 815-837

13. $\mathrm{Hu} \mathrm{Y}$. and Nualart D. Renormalized self-intersection local time for fractional Brownian motion. Ann Probab, 2005, 33: 948-983

14. Imkeller P., Perez-Abreu V. and Vives J. Chaos expansion of double intersection local time of Brownian motion in $\mathbb{R}^{d}$ and renormalization. Stoch Process Appl, 1995,56: 1-34

15. Imkeller P. and Weisz, F. The asymptotic behaviour of local times and occupation integrals of the $N$-parameter Wiener process in $\mathbb{R}^{d}$. Probab Th Rel Fields, 1994, 98: $47-75$ 
16. Imkeller P. and Weisz F. Critical dimensions for the existence of self-intersection local times of the Brownian sheet in $\mathbb{R}^{d}$. In: Seminar on Stochastic Analysis, Random Fields and Applications (Ascona, 1993), pp. 151-168, Progr. Probab., 36, Birkhäuser, Basel, 1995.

17. Imkeller P. and Weisz F. Critical dimensions for the existence of self-intersection local times of the $N$ parameter Brownian motion in $\mathbb{R}^{d}$. J Theoret Probab, 1999, 12: 721-737

18. Jiang Y. and Wang Y. Self-intersection local times and collision local times of bifractional Brownian motions. Sci China Math, 2009, 52: 1905-1919

19. Meyer P. A. Quantum for probabilists. In Lecture Notes in Math, 1538, Heidelberg: Springer, 1993

20. Mueller C. and Tribe R. Hitting properties of a random string. Electronic J Probab, 2002,7: $1-29$

21. Nualart D. The Malliavin Calculus and Related Topics. New York: Springer, 2006

22. Nualart D. and Vives J. Chaos expansion and local time. Publ Mat, 1992, 36: 827-836

23. Pitt L. P. Local times for Gaussian vector fields. Indiana Univ Math J, 1978, 27: 309330

24. Shen, G. and Yan, L. Smoothness for the collision local times of bifractional Brownian motions. Sci China Math, 2011, 54: 1859-1873

25. Shen, G., Yan, L. and Chen, C. Smoothness for the collision local time of two multidimensional bifractional Brownian motions. Czechoslovak Math J, 2012, 62: 969-989

26. Tudor C. and Xiao Y. Sample paths of the solution to the fractional-colored stochastic heat equation. Submitted, 2015

27. Watanabe S. Stochastic Differential Equation and Malliavian Calculus. Tata Institute of Fundamental Research, Springer, 1984

28. $\mathrm{Wu} \mathrm{D}$. and Xiao Y. Fractal properties of the random string process. IMS Lecture Notes-Monograph Series-High Dimensional Probability, 2006, 51: 128-147

29. Wu D. and Xiao Y. Geometric properties of the images of fractional Brownian sheets. J Fourier Anal Appl, 2007, 13: 1-37

30. Wu D. and Xiao Y. Regularity of intersection local times of fractional Brownian motions. J Thoret Probab, 2010, 23: 972-1001

31. Wu D. and Xiao Y. On local times of anisotropic Gaussian random fields. Comm Stoch Anal, 2011, 5: 15-39

32. Xiao Y. Sample path properties of anisotropic Gaussian random fields. In A Minicourse on Stochastic Partial Differential Equations, (D. Khoshnevisan and F. Rassoul-Agha, editors), Lecture Notes in Math, 1962: 145-212. New York: Springer, 2009

33. Xiao Y. and Zhang T. Local times of fractional Brownian sheets. Probab Th Rel Fields, 2002, 124: 204-226

34. Yan L., Liu J. and Chen C. On the collision local time of bifractional Brownian motions. Stoch Dyn, 2009, 9: 479-91

35. Yan L. and Shen G. On the collision local time of sub-fractional Brownian motions. Statist Probab Lett, 2010, 80: 296-308 